\title{
ESSENTIAL VARIATIONAL POISSON COHOMOLOGY
}

\author{
ALBERTO DE SOLE, VICTOR G. KAC
}

\begin{abstract}
In our recent paper DSK11 we computed the dimension of the variational Poisson cohomology $\mathcal{H}_{K}^{\bullet}(\mathcal{V})$ for any quasiconstant coefficient $\ell \times \ell$ matrix differential operator $K$ of order $N$ with invertible leading coefficient, provided that $\mathcal{V}$ is a normal algebra of differential functions over a linearly closed differential field. In the present paper we show that, for $K$ skewadjoint, the $\mathbb{Z}$-graded Lie superalgebra $\mathcal{H}_{K}^{\bullet}(\mathcal{V})$ is isomorphic to the finite dimensional Lie superalgebra $\widetilde{H}(N \ell, S)$. We also prove that the subalgebra of "essential" variational Poisson cohomology, consisting of classes vanishing on the Casimirs of $K$, is zero. This vanishing result has applications to the theory of bi-Hamiltonian structures and their deformations. At the end of the paper we consider also the translation invariant case.
\end{abstract}

\section{INTRODUCTION}

The $\mathbb{Z}$-graded Lie superalgebra $W^{\mathrm{var}}(\Pi \mathcal{V})=\bigoplus_{k=-1}^{\infty} W_{k}^{\mathrm{var}}$ of variational polyvector fields is a very convenient framework for the theory of integrable Hamiltonian PDE's. This Lie superalgebra is associated to an algebra of differential functions $\mathcal{V}$, which is an extension of the algebra of differential polynomials $R_{\ell}=\mathcal{F}\left[u_{i}^{(n)} \mid i=1, \ldots, \ell ; n \in \mathbb{Z}_{+}\right]$over a differential field $\mathcal{F}$ with the derivation $\partial$ extended to $R_{\ell}$ by $\partial u_{i}^{(n)}=u_{i}^{(n+1)}$.

The first three pieces, $W_{k}^{\mathrm{var}}$ for $k=-1,0,1$, are identified with the most important objects in the theory of integrable systems: First, $W_{-1}^{\mathrm{var}}=$ $\Pi(\mathcal{V} / \partial \mathcal{V})$, where $\mathcal{V} / \partial \mathcal{V}$ is the space of Hamiltonian functions (or local functionals), and where $\Pi$ is just to remind that it should be considered as an odd subspace of $W^{\operatorname{var}}(\Pi \mathcal{V})$. Second, $W_{0}^{\text {var }}$ is the Lie algebra of evolutionary vector fields

$$
X_{P}=\sum_{i=1}^{\ell} \sum_{n=0}^{\infty}\left(\partial^{n} P_{i}\right) \frac{\partial}{\partial u_{i}^{(n)}}, P \in \mathcal{V}^{\ell},
$$

which we identify with $\mathcal{V}^{\ell}$. Third, $W_{1}^{\text {var }}$ is identified with the space of skewadjoint $\ell \times \ell$ matrix differential operators over $\mathcal{V}$ endowed with odd parity.

Dipartimento di Matematica, Università di Roma "La Sapienza", 00185 Roma, Italy. desole@mat.uniroma1.it Supported by a PRIN grant and Fondi Ateneo, from the University of Rome.

Department of Mathematics, M.I.T., Cambridge, MA 02139, USA. kac@math.mit.edu Supported in part by an NSF grant. 
For $\int f, \int g \in W_{-1}^{\mathrm{var}}, X, Y \in W_{0}^{\mathrm{var}}$, and $H=H(\partial) \in W_{1}^{\mathrm{var}}$, the commutators are defined as follows (as usual, $\int$ denotes the canonical map $\mathcal{V} \rightarrow \mathcal{V} / \partial \mathcal{V})$

$$
\begin{aligned}
{\left[\int f, \int g\right] } & =0 \\
{\left[X, \int f\right] } & =\int X(f) \\
{[X, Y] } & =X Y-Y X \\
{\left[H, \int f\right] } & =H(\partial) \frac{\delta f}{\delta u}, \\
{\left[X_{P}, H\right] } & =X_{P}(H(\partial))-D_{P}(\partial) \circ H(\partial)-H(\partial) \circ D_{P}^{*}(\partial) .
\end{aligned}
$$

Here $\frac{\delta}{\delta u}$ is the variational derivative (see (3.4)), $D_{P}$ is the Frechet derivative (see (3.7) ), and $D^{*}(\partial)$ denotes the matrix differential operator adjoint to $D(\partial)$.

The formula for the commutator of two elements $K, H$ of $W_{1}^{\text {var }}$ (the so called Schouten bracket) is more complicated (see (3.17), but one needs only to know that conditions $[K, K]=0,[H, H]=0$ means that these matrix differential operators are Hamiltonian, and the condition $[K, H]=0$ means that they are compatible.

There have been various various versions of the notion of variational polyvector fields, but Kup80 is probably the earliest reference.

The basic notions of the theory of integrable Hamiltonian equations can be easily described in terms of the Lie superalgebra $W^{\operatorname{var}}(\Pi \mathcal{V})$. Given a Hamiltonian operator $H$ and a Hamiltonian function $\int h \in \mathcal{V} / \partial \mathcal{V}$, the corresponding Hamiltonian equation is

$$
\frac{d u}{d t}=\left[H, \int h\right], u=\left(u_{1}, \ldots, u_{\ell}\right) .
$$

One says that two Hamiltonian functions $\int h_{1}$ and $\int h_{2}$ are in involution if

$$
\left[\left[H, \int h_{1}\right], \int h_{2}\right]=0 \text {. }
$$

(Note that the LHS of (1.7) is skewasymmetric in $\int h_{1}$ and $\int h_{2}$, since both are odd elements of the Lie superalgebra $\left.W^{\operatorname{var}}(\Pi \mathcal{V})\right)$. Any $\int h_{1}$ which is in involution with $\int h$ is called an integral of motion of the Hamiltonian equation (1.6), and this equation is called integrable if there exists an infinite dimensional subspace $\Omega$ of $\mathcal{V} / \partial \mathcal{V}$ containing $\int h$ such that all elements of $\Omega$ are in involution. In this case we obtain a hierarchy of compatible integrable Hamiltonian equations, labeled by elements $\omega \in \Omega$ :

$$
\frac{d u}{d t_{\omega}}=[H, \omega] \text {. }
$$

The basic device for proving integrability of a Hamiltonian equation is the so called Lenard-Magri scheme, proposed by Lenard in early 1970's (unpublished), with an important imput by Magri Mag78. A survey of related results up to early 1990's can be found in Dor93, and a discussion in terms of Poisson vertex algebras can be found in BDSK09]. 
The Lenard-Magri scheme requires two compatible Hamiltonian operators $H$ and $K$ and a sequence of Hamiltonian functions $\int h_{n}, n \in \mathbb{Z}_{+}$, such that

$$
\left[H, \int h_{n}\right]=\left[K, \int h_{n+1}\right], n \in \mathbb{Z}_{+} .
$$

Then it is a trivial exercise in Lie superalgebra to show that all Hamiltonian functions $\int h_{n}$ are in involution (hint: use the parenthetical remark after (1.7)). Note to solve exercise one only uses the fact that $K, H$ lie in $W_{1}^{\text {var }}$, but in order to construct the sequence $\int h_{n}, n \in \mathbb{Z}_{+}$, one needs the Hamiltonian property of $H$ and $K$ and their compatibility.

The appropriate language here is the cohomological one. Since $[K, K]=0$ and $K$ is an (odd) element of $W_{1}^{\mathrm{var}}$, it follows that we have a cohomology complex

$$
\left(W^{\operatorname{var}}(\Pi \mathcal{V})=\bigoplus_{k \geq-1} W_{k}^{\operatorname{var}}, \operatorname{ad} K\right)
$$

called the variational Poisson cohomology complex. As usual, let $\mathcal{Z}_{K}^{\bullet}(\mathcal{V})=$ $\bigoplus_{k \geq-1} \mathcal{Z}_{K}^{-}$be the subalgebra of closed elements $(=\operatorname{Ker}(\operatorname{ad} K))$, and let $\mathcal{B}_{K}^{\bullet}(\mathcal{V})=\bigoplus_{k \geq-1} \mathcal{B}_{K}^{-}$be its ideal of exact elements $(=\operatorname{Im}(\operatorname{ad} K))$. Then the variational Poisson cohomology

$$
\mathcal{H}_{K}^{\bullet}(\mathcal{V})=\mathcal{Z}_{K}^{\bullet}(\mathcal{V}) / \mathcal{B}_{K}^{\bullet}(\mathcal{V})=\bigoplus_{k \geq-1} \mathcal{H}_{K}^{k},
$$

is a $\mathbb{Z}$-graded Lie superalgebra. (For usual polyvector fields the corresponding Poisson cohomology was introduced in [Lic77]; cf. [DSK11]).

Now we can try to find a solution to (1.8) by induction on $n$ as follows (see [Kra88] and [Olv87]). Since $[K, H]=0$, we have, by the Jacobi identity:

$$
\left[K,\left[H, \int h_{n}\right]\right]=-\left[H,\left[K, \int h_{n}\right]\right],
$$

hence, by the inductive assumption, the RHS of (1.9) is - $\left[H,\left[H, \int h_{n+1}\right]\right]$, which is zero since $[H, H]=0$ and $H$ is odd. Thus, $\left[H, \int h_{n}\right] \in \mathcal{Z}_{K}^{0}$. To complete the $n$-th step of induction we need that this element is exact, i.e. it equals $\left[H, \int h_{n+1}\right]$ for some $\int h_{n+1}$. But in general we have

$$
\left[H, \int h_{n}\right]=\left[K, \int h_{n+1}\right]+z_{n+1},
$$

where $z_{n+1} \in \mathcal{Z}_{K}^{0}$ only depends on the cohomology class in $\mathcal{H}_{K}^{0}$.

The best place to start the Lenard-Magri scheme is to take $\int h_{0}=C_{0} \mathcal{Z}_{K}^{-1}$, a central element for $K$. Then the first step of the Lenard-Magri scheme requires the existence of $\int h_{1}$ such that

$$
\left[H, C_{0}\right]=\left[K, \int h_{1}\right] \text {. }
$$

Taking bracket of both sides of (1.11) with arbitrary $C_{1} \in \mathcal{Z}_{K}^{-1}$, we obtain

$$
\left[\left[H, C_{0}\right], C_{1}\right]=0 \text {. }
$$

Thus, if we wish the Lenard-Magri scheme to work starting with an arbitrary central element $C_{0}$ for $K$, the Hamiltonian operator $H$ (which lies in $\mathcal{Z}_{K}^{1}$ ), must satisfy (1.12) for any $C_{0}, C_{1} \in \mathcal{Z}_{K}^{-1}$. In other words, $H$ must be "essentially closed". 
It was remarked in DMS05 that condition (1.12) is an obstruction to triviality of deformations of the Hamiltonian operator $K$, which is, of course, another important reason to be interested in "essential" variational Poisson cohomology.

We define the subalgebra $\mathcal{E Z}_{K}^{\bullet}(\mathcal{V})=\bigoplus_{k>-1} \mathcal{E Z}_{K}^{k} \subset \mathcal{Z}_{K}^{\bullet}(\mathcal{V})$ of essentially closed elements, by induction on $k \geq-1$, as follows:

$$
\mathcal{E Z}_{K}^{-1}=0, \mathcal{E} \mathcal{Z}_{K}^{k}=\left\{z \in \mathcal{Z}_{K}^{k} \mid\left[z, \mathcal{Z}_{K}^{-1}\right] \subset \mathcal{E Z}^{k-1}\right\}, k \in \mathbb{Z}_{+} .
$$

It is immediate to see that exact elements are essentially closed, and we define the essential variational Poisson cohomology as

$$
\mathcal{E H}_{K}^{\bullet}(\mathcal{V})=\mathcal{E Z}_{K}^{\bullet}(\mathcal{V}) / \mathcal{B}_{K}^{\bullet}(\mathcal{V})
$$

The first main result of the present paper is Theorem 4.3, which asserts that $\mathcal{E H}_{K}^{\bullet}(\mathcal{V})=0$, provided that $K$ is an $\ell \times \ell$ matrix differential operator of order $N$ with coefficients in $\operatorname{Mat}_{\ell \times \ell}(\mathcal{F})$ and invertible leading coefficient, that the differential field $\mathcal{F}$ is linearly closed, and that the algebra of differential functions $\mathcal{V}$ is normal. Recall that a differential field $\mathcal{F}$ is called linearly closed DSK11] if any linear inhomogenous (respectively homogenous) differential equation of order greater than or equal to 1 with coefficients in $\mathcal{F}$ has a solution (resp. nonzero solution) in $\mathcal{F}$.

The proof of Theorem 4.3 relies on our previous paper [DSK11, where, under the same assumptions on $K, \mathcal{F}$ and $\mathcal{V}$, we prove that $\operatorname{dim}_{\mathcal{C}}\left(\mathcal{H}_{K}^{k}\right)=$ $\left(\begin{array}{c}N \ell \\ k+2\end{array}\right)$, where $\mathcal{C} \subset \mathcal{F}$ is the subfield of constants, and we constructed explicit representatives of cohomology classes.

In turn, Theorem 4.3 allows us to compute the Lie superalgebra structure of $\mathcal{H}_{K}^{\bullet}(\mathcal{V})$, which is our second main result. Namely, Theorem 3.6 asserts that the $\mathbb{Z}$-graded Lie superalgebra $\mathcal{H}_{K}^{\bullet}(\mathcal{V})$ is isomorphic to the finite dimensional $\mathbb{Z}$-graded Lie superalgebra $\widetilde{H}(N \ell, S)$, of Hamiltonian vector fields over the Grassman superalgebra in $N \ell$ indeterminates $\left\{\xi_{i}\right\}_{i=1}^{N \ell}$, with Poisson bracket $\left\{\xi_{i}, \xi_{j}\right\}=s_{i j}$, divided by the central ideal $\mathcal{C} 1$, where $S=\left(s_{i j}\right)$ is a nondegenerate symmetric $N \ell \times N \ell$ matrix over $\mathcal{C}$.

We hope that Theorem 4.3 will allow further progress in the study of the Lenard-Magri scheme (work in progress). First, it leads to classification of Hamiltonian operators $H$ compatible to $K$, using techniques and results from DSKW10]. Second, it shows that if the elements $z_{n+1}$ in (1.10) are essentially closed, then they can be removed.

Also, of course, Theorem 4.3 shows that, if (1.12) holds for a Hamiltonian operator obtained by a formal deformation of $K$, then this formal deformation is trivial.

In conclusion of the paper we discuss the other "extreme" - the translation invariant case - when $\mathcal{F}=\mathcal{C}$. In this case, we give an upper bound for the dimension of $\mathcal{H}_{K}^{k}$, for an arbitrary Hamiltonian operator $K$ with coefficients in $\operatorname{Mat}_{\ell \times \ell}(\mathcal{C})$ and invertible leading coefficient, and we show that this bound is sharp if and only if $K=K_{1} \partial$, where $K_{1}$ is a symmetric nondegenerate matrix over $\mathcal{C}$. Since any Hamiltonian operator of hydrodymanic type can 
be brought, by a change of variables, to this form, our result generalizes the results of [LZ11, LZ11pr on $K$ of hydrodynamic type. Furthermore, for such operators $K$ we also prove that the essential variational Poisson cohomology is trivial, and we find a nice description of the $\mathbb{Z}$-graded Lie superalgebra $\mathcal{H}_{K}^{\bullet}$.

We are grateful to Tsinghua University and the Mathematical Sciences Center (MSC), Beijing, where this paper was written, for their hospitality, and especially Youjin Zhang and Si-Qi Liu for enlightening lectures and discussions. We also thank the Center of Mathematics and Theoretical Physics (CMTP), Rome, for continuing encouragement and support.

\section{Transitive $\mathbb{Z}$-GRAded Lie Superalgebras And PROLOnGATIONS}

Recall [GS64, Kac77] that a $\mathbb{Z}$-graded Lie superalgebra $\mathfrak{g}=\bigoplus_{k \geq-1} \mathfrak{g}_{k}$ is called transitive if any $a \in \mathfrak{g}_{k}, k \geq 0$, such that $\left[a, \mathfrak{g}_{-1}\right]=0$, is zero. Two equivalent definitions are as follows:

(i) There are no nonzero ideals of $\mathfrak{g}$ contained in $\bigoplus_{k>0} \mathfrak{g}_{k}$.

(ii) If $a \in \mathfrak{g}_{k}$ is such that $\left[\ldots\left[\left[a, C_{0}\right], C_{1}\right], \ldots, C_{k}\right]=0$ for all $C_{0}, \ldots, C_{k} \in$ $\mathfrak{g}_{-1}$, then $a=0$.

If a $\mathbb{Z}$-graded Lie superalgebra $\mathfrak{g}=\bigoplus_{k \geq-1} \mathfrak{g}_{k}$ is transitive, the Lie subalgebra $\mathfrak{g}_{0}$ acts faithfully on $\mathfrak{g}_{-1}$, hence we have an embedding $\mathfrak{g}_{0} \rightarrow g l\left(\mathfrak{g}_{-1}\right)$.

Given a Lie algebra $\mathfrak{g}$ acting faithfully on a purely odd vector superspace $U$, one calls a prolongation of the pair $(U, \mathfrak{g})$ any transitive $\mathbb{Z}$-graded Lie superalgebra $\mathfrak{g}=\bigoplus_{k>-1} \mathfrak{g}_{k}$ such that $\mathfrak{g}_{-1}=U, \mathfrak{g}_{0}=\mathfrak{g}$, and the Lie bracket between $\mathfrak{g}_{0}$ and $\mathfrak{g}_{-1}$ is given by the action of $\mathfrak{g}$ on $U$. The full prolongation of the pair $(U, \mathfrak{g})$ is a prolongation containing any other prolongation of $(U, \mathfrak{g})$. It always exists and is unique.

2.1. The $\mathbb{Z}$-graded Lie superalgebra $W(n)$. Let $\Lambda(n)$ be the Grassman superalgebra over the field $\mathcal{C}$ on odd generators $\xi_{1}, \ldots, \xi_{n}$. Let $W(n)$ be the Lie superalgebra of all derivations of the superalgebra $\Lambda(n)$, with the following $\mathbb{Z}$-grading: for $k \geq-1, W_{k}(n)$ is spanned by derivations of the form $\xi_{i_{1}} \ldots \xi_{i_{k+1}} \frac{\partial}{\partial \xi_{j}}$. In particular, $W_{-1}(n)=\left\langle\frac{\partial}{\partial \xi_{i}}\right\rangle_{i=1}^{n}=\Pi \mathcal{C}^{n}$, and $W_{0}(n)=\left\langle\xi_{i} \frac{\partial}{\partial \xi_{j}}\right\rangle_{i, j=1}^{n} \simeq g l(n)$. It is easy to see that $W(n)$ is the full prolongation of $\left(\Pi \mathcal{C}^{n}, g l(n)\right)$ Kac77]. Consequently, any transitive $\mathbb{Z}$-graded Lie superalgebra $\mathfrak{g}=\bigoplus_{k \geq-1} \mathfrak{g}_{k}$, with $\operatorname{dim}_{\mathcal{C}} \mathfrak{g}_{-1}=n$, embeds in $W(n)$.

2.2. The $\mathbb{Z}$-graded Lie superalgebra $\widetilde{H}(n, S)$. Let $S=\left(s_{i j}\right)_{i, j=1}^{n}$ be a symmetric $n \times n$ matrix over $\mathcal{C}$. Consider the following subalgebra of the Lie algebra $g l(n)$ :

$$
\text { so }(n, S)=\left\{A \in \operatorname{Mat}_{n \times n}(\mathcal{C}) \mid A^{T} S+S A=0, \operatorname{Tr}(A)=0\right\} .
$$

We endow the Grassman superalgebra $\Lambda(n)$ with a structure of a Poisson superalgebra by letting $\left\{\xi_{i}, \xi_{j}\right\}_{S}=s_{i j}$. A closed formula for the Poisson 
bracket on $\Lambda(n)$ is

$$
\{f, g\}_{S}=(-1)^{p(f)+1} \sum_{i, j=1}^{n} s_{i j} \frac{\partial f}{\partial \xi_{i}} \frac{\partial g}{\partial \xi_{j}} .
$$

We introduce a $\mathbb{Z}$-grading of the superspace $\Lambda(n)$ by letting $\operatorname{deg}\left(\xi_{i_{1}} \ldots \xi_{i_{s}}\right)=$ $s-2$. Note that this is a Lie superalgebra $\mathbb{Z}$ grading $\Lambda(n)=\bigoplus_{k=-2}^{n-2} \Lambda_{k}(n)$ (but it is not an associative superalgebra grading). Note also that $\Lambda_{-2}(n)=$ $\mathcal{C} 1 \subset \Lambda(n)$ is a central ideal of this Lie superalgebra. Hence $\Lambda(n) / \mathcal{C} 1$ inherits the structure of a $\mathbb{Z}$-graded Lie superalgebra of dimension $2^{n}-1$, which we denote by $\widetilde{H}(n, S)=\bigoplus_{k=-1}^{n-2} \widetilde{H}_{k}(n, S)$.

The -1-st degree subspace is $\widetilde{H}_{-1}(n, S)=\left\langle\xi_{i}\right\rangle_{i=1}^{n} \simeq \Pi \mathcal{C}^{n}$, and the 0 -th degree subspace $\widetilde{H}_{0}(n, S)=\left\langle\xi_{i} \xi_{j}\right\rangle_{i, j=1}^{n}$ is a Lie subalgebra of dimension $\left(\begin{array}{l}n \\ 2\end{array}\right)$.

Identifying $\widetilde{H}_{-1}(n, S)$ with $\Pi \mathcal{C}^{n}$ (using the basis $\xi_{i}, i=1, \ldots, n$ ) and $\widetilde{H}_{0}(n, S)$ with the space of skewsymmetric $n \times n$ matrices over $\mathcal{C}$ (via $\xi_{i} \xi_{j} \mapsto$ $\left.\left(E_{i j}-E_{j i}\right) / 2\right)$, the action of $\widetilde{H}_{0}(n, S)$ on $\widetilde{H}_{-1}(n, S)$ becomes: $\{A, v\}_{S}=A S v$. Note that, if $A$ is skewsymmetric, then $A S$ lies in $s o(n, S)$. Hence, we have a homomorphism of Lie superalgebras:

$$
\widetilde{H}_{-1}(n, S) \oplus \widetilde{H}_{0}(n, S) \rightarrow \Pi \mathcal{C}^{n} \oplus \operatorname{so}(n, S), \quad(v, A) \mapsto(v, A S) .
$$

Lemma 2.1. The map (2.2) is bijective if and only if $S$ has rank $n$ or $n-1$.

Proof. Clearly, if $S$ is nondegenerate, the map (2.2) is bijective. Moreover, if $S$ has rank less than $n-1$, the map (2.2) is clearly not injective. In the remaining case when $S$ has rank $n-1$, we can assume it has the form

$$
S=\left(\begin{array}{cc}
0 & 0 \\
0 & T
\end{array}\right)
$$

where $T$ is a nondegenerate symmetric $(n-1) \times(n-1)$ matrix. In this case, one immediately checks that the map (2.2) is injective. Moreover,

$$
s o(n, S)=\left\{\left(\begin{array}{cc}
0 & B^{T} \\
0 & A
\end{array}\right) \mid B \in \mathcal{C}^{\ell}, A \in \operatorname{so}(n-1, T)\right\} .
$$

Hence, $\operatorname{dim}_{\mathcal{C}} \operatorname{so}(n, S)=n-1+\left(\begin{array}{c}n-1 \\ 2\end{array}\right)=\left(\begin{array}{l}n \\ 2\end{array}\right)=\operatorname{dim}_{\mathcal{C}} \widetilde{H}_{0}(n, S)$.

Proposition 2.2. If $S$ has rank $n$ or $n-1$, then $\widetilde{H}(n, S)$ is the full prolongation of the pair $\left(\mathcal{C}^{n}, \operatorname{so}(n, S)\right)$.

Proof. For $S$ nondegenerate, the proof is can be found in Kac77. We reduce below the case $r k(S)=n-1$ to the case of nondegenerate $S$. If $\operatorname{rk}(S)=\ell=n-1$, we can choose a basis $\left\langle\eta, \xi_{1}, \ldots, \xi_{\ell}\right\rangle$, such that the matrix $S$ is of the form (2.3). Define the map $\varphi_{S}: \widetilde{H}(n, S) \rightarrow W(n)$, given by

$$
\begin{aligned}
& \varphi_{S}\left(f\left(\xi_{1}, \ldots, \xi_{\ell}\right)\right)=\{f, \cdot\}_{S}=(-1)^{p(f)+1} \sum_{i, j=1}^{\ell} t_{i j} \frac{\partial f}{\partial \xi_{i}} \frac{\partial}{\partial \xi_{j}}, \\
& \varphi_{S}\left(f\left(\xi_{1}, \ldots, \xi_{\ell}\right) \eta\right)=f\left(\xi_{1}, \ldots, \xi_{\ell}\right) \frac{\partial}{\partial \eta}
\end{aligned}
$$


It is easy to check that $\varphi_{S}$ is an injective homomorphism of $\mathbb{Z}$-graded Lie superalgebras. Hence, we can identify $\widetilde{H}(n, S)$ with its image in $W(n)$.

Since $\varphi_{S}\left(\widetilde{H}_{-1}(n, S)\right)=\Pi \mathcal{C}^{n}=W_{-1}(n)$, the $\mathbb{Z}$-graded Lie superalgebra $\varphi_{S}(\widetilde{H}(n, S))$ (hence $\left.\widetilde{H}(n, S)\right)$ is transitive. It remains to prove that it is the full prolongation of the pair $\left(\widetilde{H}_{-1}(n, S), \widetilde{H}_{0}(n, S)\right)$. For this, we will prove that, if

$$
X=f_{0} \frac{\partial}{\partial \eta}+\sum_{i=1}^{\ell} f_{i} \frac{\partial}{\partial \xi_{i}} \in W_{k}(n),
$$

with $f_{i} \in \Lambda(n)$, homogenous polynomials of degree $k+1 \geq 2$, is such that

$$
\left[\frac{\partial}{\partial \eta}, X\right], \quad\left[\frac{\partial}{\partial \xi_{i}}, X\right] \in \varphi_{S}\left(\widetilde{H}_{k-1}(n, S)\right) \quad \forall i=1, \ldots \ell,
$$

then $X \in \varphi_{S}\left(\widetilde{H}_{k}(n, S)\right)$. Conditions (2.5) imply that all $f_{0}, \ldots, f_{\ell}$ are polynomials in $\xi_{1}, \ldots, \xi_{\ell}$ only, and there exist $g_{1}, \ldots, g_{\ell}$, polynomials in $\xi_{1}, \ldots, \xi_{\ell}$, such that

$$
\frac{\partial f_{j}}{\partial \xi_{i}}=(-1)^{p\left(g_{i}\right)+1} \sum_{k=1}^{\ell} t_{j k} \frac{\partial g_{i}}{\partial \xi_{k}},
$$

for every $i, j \in\{1, \ldots \ell\}$. On the other hand, the condition that $X \in$ $\varphi_{S}\left(\widetilde{H}_{k}(n, S)\right)$ means that there exists $h$, a polynomial in $\xi_{1}, \ldots, \xi_{\ell}$, such that

$$
f_{i}=(-1)^{p(h)+1} \sum_{k=1}^{\ell} t_{i k} \frac{\partial h}{\partial g_{k}} .
$$

To conclude, we observe that conditions (2.6) imply the existence of $h$ solving equation (2.7), since $\widetilde{H}(\ell, T)$ is a full prolongation.

Remark 2.3. The notation $\widetilde{H}(n, S)$ comes from the fact that, if $S$ is nondegenerate, then the derived Lie superalgebra $H(n, S)=\{\widetilde{H}(n, S), \widetilde{H}(n, S)\}=$ $\bigoplus_{k=-1}^{n-3} \widetilde{H}_{k}(n, S)$ has codimension 1 in $\widetilde{H}(n, S)$, and it is simple for $n \geq 4$.

\section{Variational Poisson COHOMOlogy}

In this section we recall our results from [DSK11] on the variational Poisson cohomology, in the notation of the present paper.

3.1. Algebras of differential functions. An algebra of differential functions $\mathcal{V}$ in one independent variable $x$ and $\ell$ dependent variables $u_{i}$, indexed by the set $I=\{1, \ldots, \ell\}$, is, by definition, a differential algebra (i.e. a unital commutative associative algebra with a derivation $\partial$ ), endowed with commuting derivations $\frac{\partial}{\partial u_{i}^{(n)}}: \mathcal{V} \rightarrow \mathcal{V}$, for all $i \in I$ and $n \in \mathbb{Z}_{+}$, such that, 
given $f \in \mathcal{V}, \frac{\partial}{\partial u_{i}^{(n)}} f=0$ for all but finitely many $i \in I$ and $n \in \mathbb{Z}_{+}$, and the following commutation rules with $\partial$ hold:

$$
\left[\frac{\partial}{\partial u_{i}^{(n)}}, \partial\right]=\frac{\partial}{\partial u_{i}^{(n-1)}},
$$

where the RHS is considered to be zero if $n=0$. An equivalent way to write the identities (3.1) is in terms of generating series:

$$
\sum_{n \in \mathbb{Z}_{+}} z^{n} \frac{\partial}{\partial u_{i}^{(n)}} \circ \partial=(z+\partial) \circ \sum_{n \in \mathbb{Z}_{+}} z^{n} \frac{\partial}{\partial u_{i}^{(n)}} .
$$

As usual we shall denote by $f \mapsto \int f$ the canonical quotient map $\mathcal{V} \rightarrow \mathcal{V} / \partial \mathcal{V}$.

We call $\mathcal{C}=\operatorname{Ker}(\partial) \subset \mathcal{V}$ the subalgebra of constant functions, and we denote by $\mathcal{F} \subset \mathcal{V}$ the subalgebra of quasiconstant functions, defined by

$$
\mathcal{F}=\left\{f \in \mathcal{V} \mid \frac{\partial f}{\partial u_{i}^{(n)}}=0 \forall i \in I, n \in \mathbb{Z}_{+}\right\} .
$$

It is not hard to show DSK11 that $\mathcal{C} \subset \mathcal{F}, \partial \mathcal{F} \subset \mathcal{F}$, and $\mathcal{F} \cap \partial \mathcal{V}=\partial \mathcal{F}$. Throughout the paper we will assume that $\mathcal{F}$ is a field of characteristic zero, hence so is $\mathcal{C} \subset \mathcal{F}$. Unless otherwise specified, all vector spaces, as well as tensor products, direct sums, and Hom's, will be considered over the field $\mathcal{C}$.

One says that $f \in \mathcal{V}$ has differential order $n$ in the variable $u_{i}$ if $\frac{\partial f}{\partial u_{i}^{(n)}} \neq 0$ and $\frac{\partial f}{\partial u_{i}^{(m)}}=0$ for all $m>n$.

The main example of an algebra of differential functions is the ring of differential polynomials over a differential field $\mathcal{F}, R_{\ell}=\mathcal{F}\left[u_{i}^{(n)} \mid i \in I, n \in \mathbb{Z}_{+}\right]$, where $\partial\left(u_{i}^{(n)}\right)=u_{i}^{(n+1)}$. Other examples can be constructed starting from $R_{\ell}$ by taking a localization by some multiplicative subset $S$, or an algebraic extension obtained by adding solutions of some polynomial equations, or a differential extension obtained by adding solutions of some differential equations.

The variational derivative $\frac{\delta}{\delta u}: \mathcal{V} \rightarrow \mathcal{V}^{\ell}$ is defined by

$$
\frac{\delta f}{\delta u_{i}}:=\sum_{n \in \mathbb{Z}_{+}}(-\partial)^{n} \frac{\partial f}{\partial u_{i}^{(n)}} .
$$

It follows immediately from (3.2) that $\partial \mathcal{V} \subset \operatorname{Ker} \frac{\delta}{\delta u}$.

A vector field is, by definition, a derivation of $\mathcal{V}$ of the form

$$
X=\sum_{i \in I, n \in \mathbb{Z}_{+}} P_{i, n} \frac{\partial}{\partial u_{i}^{(n)}}, \quad P_{i, n} \in \mathcal{V} .
$$

We denote by $\operatorname{Vect}(\mathcal{V})$ the Lie algebra of all vector fields. A vector field $X$ is called evolutionary if $[\partial, X]=0$, and we denote by $\operatorname{Vect}^{\partial}(\mathcal{V}) \subset \operatorname{Vect}(\mathcal{V})$ 
the Lie subalgebra of all evolutionary vector fields. By (3.1), a vector field $X$ is evolutionary if and only if it has the form

$$
X_{P}=\sum_{i \in I, n \in \mathbb{Z}_{+}}\left(\partial^{n} P_{i}\right) \frac{\partial}{\partial u_{i}^{(n)}},
$$

where $P=\left(P_{i}\right)_{i \in I} \in \mathcal{V}^{\ell}$, is called the characteristic of $X_{P}$.

Given $P \in \mathcal{V}^{\ell}$, we denote by $D_{P}=\left(\left(D_{P}\right)_{i j}(\partial)\right)_{i, j \in I}$ its Frechet derivative, given by

$$
\left(D_{P}\right)_{i j}(\partial)=\sum_{n \in \mathbb{Z}_{+}} \frac{\partial P_{i}}{\partial u_{j}^{(n)}} \partial^{n}
$$

Recall from BDSK09] that an algebra of differential functions $\mathcal{V}$ is called normal if we have $\frac{\partial}{\partial u_{i}^{(m)}}\left(\mathcal{V}_{m, i}\right)=\mathcal{V}_{m, i}$ for all $i \in I, m \in \mathbb{Z}_{+}$, where we let

$$
\mathcal{V}_{m, i}:=\left\{f \in \mathcal{V} \mid \frac{\partial f}{\partial u_{j}^{(n)}}=0 \text { if }(n, j)>(m, i) \text { in lexicographic order }\right\} .
$$

We also denote $\mathcal{V}_{m, 0}=\mathcal{V}_{m-1, \ell}$, and $\mathcal{V}_{0,0}=\mathcal{F}$.

The algebra $R_{\ell}$ is obviously normal. Moreover, any its extension $\mathcal{V}$ can be further extended to a normal algebra. Conversely, it is proved in [DSK09] that any normal algebra of differential functions $\mathcal{V}$ is automatically a differential algebra extension of $R_{\ell}$. Throughout the paper we shall assume that $\mathcal{V}$ is an extension of $R_{\ell}$.

Recall also from DSK11] that a differential field $\mathcal{F}$ is called linearly closed if any linear differential equation,

$$
a_{n} u^{(n)}+\cdots+a_{1} u^{\prime}+a_{0} u=b,
$$

with $n \geq 0, a_{0}, \ldots, a_{n} \in \mathcal{F}, a_{n} \neq 0$, has a solution in $\mathcal{F}$ for every $b \in \mathcal{F}$, and it has a nonzero solution for $b=0$, provided that $n \geq 1$.

3.2. The universal Lie superalgebra $W^{\operatorname{var}}(\Pi \mathcal{V})$ of variational polyvector fields. Recall the definition of the universal Lie superalgebra of variational polyvector fields $W^{\mathrm{var}}(\Pi \mathcal{V})$, associated to the algebra of differential funtions $\mathcal{V}$ [DSK11]. We let

$$
W^{\mathrm{var}}(\Pi \mathcal{V})=\bigoplus_{k=-1}^{\infty} W_{k}^{\mathrm{var}}
$$

where $W_{k}^{\mathrm{var}}$ is the superspace of parity $k \bmod 2$ consisting of all skewsymmetric arrays, i.e. arrays of polynomials

$$
P=\left(P_{i_{0}, \ldots, i_{k}}\left(\lambda_{0}, \ldots, \lambda_{k}\right)\right)_{i_{1}, \ldots, i_{k} \in I},
$$

where $P_{i_{0}, \ldots, i_{k}}\left(\lambda_{0}, \ldots, \lambda_{k}\right) \in \mathcal{V}\left[\lambda_{0}, \ldots, \lambda_{k}\right] /\left(\partial+\lambda_{0}+\cdots+\lambda_{k}\right)$ are skewsymmetric with respect to simultaneous permutations of the variables $\lambda_{0}, \ldots, \lambda_{k}$ and the indices $i_{0}, \ldots, i_{k}$. By $\mathcal{V}\left[\lambda_{0}, \ldots, \lambda_{k}\right] /\left(\partial+\lambda_{0}+\cdots+\lambda_{k}\right)$ we mean the quotient of the space $\mathcal{V}\left[\lambda_{0}, \ldots, \lambda_{k}\right]$ by the image of the operator $\partial+\lambda_{0}+\cdots+\lambda_{k}$. 
Clearly, for $k=-1$ this space is $\mathcal{V} / \partial \mathcal{V}$ and, for $k \geq 0$, we can identify it with the algebra of polynomials $\mathcal{V}\left[\lambda_{0}, \ldots, \lambda_{k-1}\right]$ by letting

$$
\lambda_{k}=-\lambda_{0}-\cdots-\lambda_{k-1}-\partial,
$$

with $\partial$ acting from the left. We then define the following $\mathbb{Z}$-graded Lie superalgebra bracket on $W^{\operatorname{var}}(\Pi \mathcal{V})$. For $P \in W_{h}^{\text {var }}$ and $Q \in W_{k-h}^{\text {var }}$, with $-1 \leq h \leq k+1$, we let $[P, Q]:=P \square Q-(-1)^{h(k-h)} Q \square P$, where $P \square Q \in W_{k}^{\text {var }}$ is zero if $h=k-h=-1$, and otherwise it is given by

$$
\begin{aligned}
& (P \square Q)_{i_{0}, \ldots, i_{k}}\left(\lambda_{0}, \ldots, \lambda_{k}\right)=\sum_{\sigma \in \mathcal{S}_{h, k}} \operatorname{sign}(\sigma) \sum_{j \in I, n \in \mathbb{Z}_{+}} \\
& P_{j, i_{\sigma(k-h+1)}, \ldots, i_{\sigma(k)}}\left(\lambda_{\sigma(0)}+\cdots+\lambda_{\sigma(k-h)}+\partial, \lambda_{\sigma(k-h+1)}, \ldots, \lambda_{\sigma(k)}\right) \rightarrow \\
& \left(-\lambda_{\sigma(0)}-\cdots-\lambda_{\sigma(k-h)}-\partial\right)^{n} \frac{\partial}{\partial u_{j}^{(n)}} Q_{i_{\sigma(0)}, \ldots, i_{\sigma(k-h)}}\left(\lambda_{\sigma(0)}, \ldots, \lambda_{\sigma(k-h)}\right),
\end{aligned}
$$

where $\mathcal{S}_{h, k}$ denotes the set of $h$-shuffles in the group $S_{k+1}=\operatorname{Perm}\{0, \ldots, k\}$, i.e. the permutations $\sigma$ satisfying

$$
\sigma(0)<\cdots<\sigma(k-h), \quad \sigma(k-h+1)<\cdots<\sigma(k) .
$$

The arrow in (3.10) means that $\partial$ should be moved to the right. Note that, by the skewsymmetry conditions on $P$ and $Q$, we can replace the sum over shuffles by the sum over the whole permutation group $S_{k+1}$, provided that we divide by $h$ ! $(k-h+1)$ !. It follows from Proposition 9.1 and the identification (9.22) in [DSK11, that the box product (3.10) is well defined and the corresponding commutator makes $W^{\text {var }}(\Pi \mathcal{V})$ into a $\mathbb{Z}$-graded Lie superalgebra.

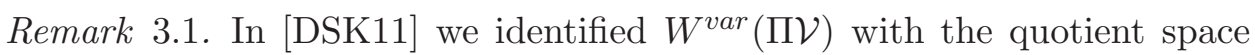
$\Omega^{\bullet}(\mathcal{V})=\widetilde{\Omega}^{\bullet}(\mathcal{V}) / \partial \widetilde{\Omega}^{\bullet}(\mathcal{V})$, where $\widetilde{\Omega}^{\bullet}(\mathcal{V})$ is the commutative associative unital superalgebra freely generated over $\mathcal{V}$ by odd generators $\theta_{i}^{(m)}=\delta u_{i}^{(m)}, i \in$ $I, m \in \mathbb{Z}_{+}$, and where $\partial: \widetilde{\Omega}^{\bullet}(\mathcal{V}) \rightarrow \widetilde{\Omega}^{\bullet}(\mathcal{V})$ extends $\partial: \mathcal{V} \rightarrow \mathcal{V}$ to an even derivation such that $\partial \theta_{i}^{(m)}=\theta_{i}^{(m+1)}$. This identification is given by mapping the array

$$
P=\left(\sum_{m_{0}, \ldots, m_{k} \in \mathbb{Z}_{+}} f_{i_{0}, \ldots, i_{k}}^{m_{0}, \ldots, m_{k}} \lambda_{0}^{m_{0}} \ldots \lambda_{k}^{m_{k}}\right)_{i_{0}, \ldots, i_{k} \in I} \in W_{k}^{v a r}
$$

to the element

$$
\int \sum_{i_{0}, \ldots, i_{k} \in I} \sum_{m_{0}, \ldots, m_{k} \in \mathbb{Z}_{+}} f_{i_{0}, \ldots, i_{k}}^{m_{0}, \ldots, m_{k}} \theta_{i_{0}}^{\left(m_{0}\right)} \ldots \theta_{i_{k}}^{\left(m_{k}\right)} \in \Omega^{k+1}(\mathcal{V}) .
$$

(It is easy to see that this map is well defined and bijective.) Here $\int$ denotes, as usual, the quotient map $\widetilde{\Omega}^{\bullet}(\mathcal{V}) \rightarrow \widetilde{\Omega}^{\bullet}(\mathcal{V}) / \partial \widetilde{\Omega}^{\bullet}(\mathcal{V})=\Omega^{\bullet}(\mathcal{V})$. We extend the variational derivative to a map

$$
\frac{\delta}{\delta u_{i}}=\sum_{n \in \mathbb{Z}_{+}}(-\partial)^{n} \circ \frac{\partial}{\partial u_{i}^{(n)}}: \Omega^{k}(\mathcal{V}) \rightarrow \Omega^{k+1}(\mathcal{V}),
$$


by letting $\frac{\partial}{\partial u_{i}^{(n)}}$ acts on coefficients $(\in \mathcal{V})$. Furthermore, we introduce the odd variational derivatives

$$
\frac{\delta}{\delta \theta_{i}}=\sum_{n \in \mathbb{Z}_{+}}(-\partial)^{n} \circ \frac{\partial}{\partial \theta_{i}^{(n)}}: \Omega^{k}(\mathcal{V}) \rightarrow \Omega^{k+1}(\mathcal{V}) .
$$

Then the box product (3.10) takes, under the identification $W^{v a r}(\Pi \mathcal{V}) \simeq$ $\Omega^{\bullet}(\mathcal{V})$, the following simple form [Get02]:

$$
P \square Q=\sum_{i \in I} \frac{\delta P}{\delta \theta_{i}} \frac{\delta Q}{\delta u_{i}} .
$$

We describe explicitly the spaces $W_{k}^{\mathrm{var}}$ for $k=-1,0,1$. Clearly, $W_{-1}^{\mathrm{var}}=$ $\mathcal{V} / \partial \mathcal{V}$. Also $W_{0}^{\mathrm{var}}=\mathcal{V}^{\ell}$ thanks to the obvious identification of $\mathcal{V}[\lambda] /(\partial+\lambda)$ with $\mathcal{V}$. Finally, the space $\mathcal{V}[\lambda, \mu] /(\partial+\lambda+\mu)$ is identified with $\mathcal{V}[\lambda] \simeq$ $\mathcal{V}[\partial]$, by letting $\mu=-\partial$ moved to the left and $\lambda=\partial$ moved to the right. Hence elements in $W_{1}^{\text {var }}$ correspond to $\ell \times \ell$ matrix differential operators over $\mathcal{V}$, and the skewsymmetry condition for an element of $W_{1}^{\text {var }}$ translates into the skewadjointness of the corresponding matrix differential operator (i.e. to the condition $H_{j i}^{*}(\partial)=-H_{i j}(\partial)$, where, as usual, for a differential operator $L(\partial)=\sum_{n} l_{n} \partial^{n}$, its adjoint is $\left.L^{*}(\partial)=\sum_{n}(-\partial)^{n} \circ l_{n}\right)$. In order to keep the same identification as in [DSK11, we associate to the array $P=$ $\left(P_{i j}(\lambda, \mu)\right)_{i, j \in I} \in W_{1}^{\mathrm{var}}$, the following skewadjoint $\ell \times \ell$ matrix differential operator $H=\left(H_{i j}(\partial)\right)_{i, j \in I}$, where

$$
H_{i j}(\lambda)=P_{j i}(\lambda,-\lambda-\partial),
$$

and $\partial$ acts from the left.

Next, we write some explicit formulas for the Lie brackets in $W^{\operatorname{var}}(\Pi \mathcal{V})$. Since $\mathcal{S}_{-1, k}=\emptyset$ and $\mathcal{S}_{k+1, k}=\{1\}$, we have, for $\int h \in \mathcal{V} / \partial \mathcal{V}=W_{-1}^{\mathrm{var}}$ and $Q \in W_{k+1}^{\mathrm{var}}:$

$$
\begin{aligned}
& {\left[\int h, Q\right]_{i_{0}, \ldots, i_{k}}\left(\lambda_{0}, \ldots, \lambda_{k}\right)=(-1)^{k}\left[Q, \int h\right]_{i_{0}, \ldots, i_{k}}\left(\lambda_{0}, \ldots, \lambda_{k}\right)} \\
& =(-1)^{k} \sum_{j \in I} Q_{j, i_{0}, \ldots, i_{k}}\left(\partial, \lambda_{0}, \ldots, \lambda_{k}\right) \rightarrow \frac{\delta h}{\delta u_{j}},
\end{aligned}
$$

In particular, $\left[\int h, \int f\right]=0$ for $\int f \in \mathcal{V} / \partial \mathcal{V}$. For $Q \in \mathcal{V}^{\ell}=W_{0}^{\text {var }}$ we have

$$
\left[Q, \int h\right]=-\left[\int h, Q\right]=\sum_{j \in I} \int Q_{j} \frac{\delta h}{\delta u_{j}}=\int X_{Q}(h),
$$

where $X_{Q}$ is the evolutionary vector field with characteristics $Q$, defined in (3.6). Furthermore, for $H=\left(H_{i j}(\partial)\right)_{i, j \in I} \in W_{1}^{\text {var }}$ (via the identification (3.11)), we have

$$
\left[H, \int h\right]=H(\partial) \frac{\delta h}{\delta u} \in \mathcal{V}^{\ell}
$$


Since $\mathcal{S}_{0, k}=\{1\}$ and $\mathcal{S}_{k, k}=\{(\alpha, 0, \stackrel{\alpha}{.}, k)\}_{\alpha=0}^{k}$, we have, for $P \in \mathcal{V}^{\ell}=$ $W_{0}^{\mathrm{var}}$ and $Q \in W_{k}^{\mathrm{var}}$,

$$
\begin{aligned}
& {[P, Q]_{i_{0}, \ldots, i_{k}}\left(\lambda_{0}, \ldots, \lambda_{k}\right)=X_{P}\left(Q_{i_{0}, \ldots, i_{k}}\left(\lambda_{0}, \ldots, \lambda_{k}\right)\right)} \\
& -\sum_{\alpha=0}^{k} \sum_{j \in I, n \in \mathbb{Z}_{+}} Q_{i_{0}, \ldots, \dot{j}, \ldots, i_{k}}\left(\lambda_{0}, \ldots, \lambda_{\alpha}+\partial, \ldots, \lambda_{k}\right)_{\rightarrow}\left(-\lambda_{\alpha}-\partial\right)^{n} \frac{\partial P_{i_{\alpha}}}{\partial u_{j}^{(n)}} .
\end{aligned}
$$

In particular, for $Q \in \mathcal{V}^{\ell}=W_{0}^{\text {var }}$, we get the usual commutator of evolutionary vector fields:

$$
[P, Q]_{i}=X_{P}\left(Q_{i}\right)-X_{Q}\left(P_{i}\right),
$$

while, for a skewadjoint $\ell \times \ell$ matrix differential operator $H(\partial) \in W_{1}^{\text {var }}$, we get

$$
[P, H](\partial)=X_{P}(H(\partial))-D_{P}(\partial) \circ H(\partial)-H(\partial) \circ D_{P}^{*}(\partial),
$$

where, in the first term of the RHS, $X_{P}(H(\partial))$ denotes the $\ell \times \ell$ matrix differential operator whose $(i, j)$ entry is obtained by applying $X_{P}$ to the coefficients of the differential operator $H_{i j}(\partial)$. In the last two terms of the RHS of (3.15), $D_{P}$ denotes the Frechet derivative of $P$, defined in (3.7), and $D_{P}^{*}$ is its adjoint matrix differential operator.

Finally, we write equation (3.10) in the case when $h=1$. Since $\mathcal{S}_{1, k}=$ $\{(0, \stackrel{\alpha}{\therefore}, k, \alpha)\}_{\alpha=0}^{k}$ and $\mathcal{S}_{k-1, k}=\left\{\left(\alpha, \beta, 0, \stackrel{\alpha}{\alpha} \stackrel{\beta}{\beta}^{\beta}, k\right)\right\}_{0 \leq \alpha<\beta \leq k}^{k}$, we have, for a skewadjoint matrix differential operator $H=\left(H_{i j}(\partial)\right)_{i, j \in I} \in W_{1}^{\text {var }}$ (via the identificatino (3.11)) and for $P \in W_{k-1}^{\mathrm{var}}$ :

$$
\begin{aligned}
& {[H, P]_{i_{0}, \ldots, i_{k}}\left(\lambda_{0}, \ldots, \lambda_{k}\right)=(-1)^{k+1} \sum_{j \in I, n \in \mathbb{Z}_{+}} \sum_{\alpha=0}^{k}(-1)^{\alpha}} \\
& \left(\frac{\partial P_{i_{0}, \stackrel{\alpha}{\alpha}, i_{k}}\left(\lambda_{0}, \stackrel{\alpha}{\alpha}, \lambda_{k}\right)}{\partial u_{j}^{(n)}}\left(\lambda_{\alpha}+\partial\right)^{n} H_{j, i_{\alpha}}\left(\lambda_{\alpha}\right)+\sum_{\beta=\alpha+1}^{k}(-1)^{\beta}\right.
\end{aligned}
$$

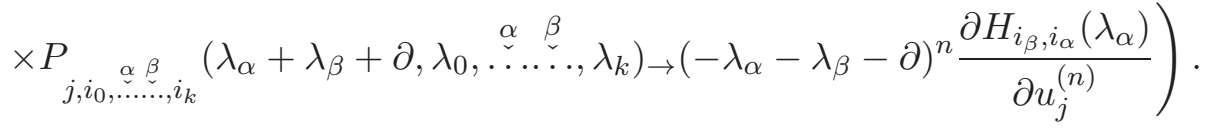

In particular, if $K=\left(K_{i j}(\partial)\right)_{i, j \in I} \in W_{1}^{\mathrm{var}}$, we have $[K, H]=[H, K]=$ $K \square H+H \square K$, where

$$
\begin{aligned}
& (K \square H)_{i_{0}, i_{1}, i_{2}}\left(\lambda_{0}, \lambda_{1}, \lambda_{2}\right)=\sum_{j \in I, n \in \mathbb{Z}_{+}}\left(\frac{\partial H_{i_{0}, i_{1}}\left(\lambda_{1}\right)}{\partial u_{j}^{(n)}}\left(\lambda_{2}+\partial\right)^{n} K_{j, i_{2}}\left(\lambda_{2}\right)\right. \\
& \left.+\frac{\partial H_{i_{1}, i_{2}}\left(\lambda_{2}\right)}{\partial u_{j}^{(n)}}\left(\lambda_{0}+\partial\right)^{n} K_{j, i_{0}}\left(\lambda_{0}\right)+\frac{\partial H_{i_{2}, i_{0}}\left(\lambda_{0}\right)}{\partial u_{j}^{(n)}}\left(\lambda_{1}+\partial\right)^{n} K_{j, i_{1}}\left(\lambda_{1}\right)\right) .
\end{aligned}
$$


Remark 3.2. Given a skewadjoint matrix differential operator $H=\left(H_{i j}(\partial)\right)$, we can define the corresponding "variational" $\lambda$-brackets $\left\{\cdot_{\lambda} \cdot\right\}_{H}: \mathcal{V} \times \mathcal{V} \rightarrow$ $\mathcal{V}[\lambda]$, given by the following formula (cf. DSK06]):

$$
\left\{f_{\lambda} g\right\}=\sum_{i, j \in I, m, n \in \mathbb{Z}_{+}} \frac{\partial g}{\partial u_{j}^{(n)}}(\lambda+\partial)^{n} H_{j i}(\lambda+\partial)(-\lambda-\partial)^{m} \frac{\partial f}{\partial u_{i}^{(m)}} .
$$

One can write the above formulas in this language (cf. [DSK11]).

Proposition 3.3. The $\mathbb{Z}$-graded Lie superalgebra $W^{\operatorname{var}}(\Pi \mathcal{V})$ is transitive, hence it is a prolongation of the pair $\left(\Pi \mathcal{V} / \partial \mathcal{V}, \operatorname{Vect}^{\partial}(\mathcal{V})\right)$.

Proof. First note that, if $H(\partial)$ is an $\ell \times \ell$ matrix differential operator such that $H(\partial) \frac{\delta f}{\delta u}=0$ for every $f \in \mathcal{V}$, then $H(\partial)=0$ (cf. [BDSK09]). Indeed, if $H(\partial)$ has order $N$ and $H_{i j}(\partial)=\sum_{n=0}^{N} h_{i j ; n} \partial^{n}$ with $h_{i j ; N} \neq 0$, then letting $f=\frac{(-1)^{M}}{2}\left(u_{j}^{(M)}\right)^{2}$, we have $\frac{\delta f}{\delta u_{k}}=\delta_{k, j} u_{j}^{(2 M)}$ and, for $M$ sufficiently large, $\frac{\partial}{\partial u_{j}^{(2 M+N)}}\left(H(\partial) \frac{\delta f}{\delta u}\right)_{i}=h_{i j ; N} \neq 0$ (here we are using the assumption that $\mathcal{V}$ contains $R_{\ell}$ ). The claim follows immediately by this observation and equation (3.12).

3.3. The cohomology complex $\left(W^{\operatorname{var}}(\Pi \mathcal{V}), \delta_{K}\right)$. Let $K=\left(K_{i j}(\partial)\right)_{i, j \in I} \in$ $W_{1}^{\text {var }}$ be a Hamiltonian operator, i.e. $K$ is skewadjoint and $[K, K]=0$. Then $(\operatorname{ad} K)^{2}=0$, and we can consider the associated variational Poisson cohomology complex $\left(W^{\operatorname{var}}(\Pi \mathcal{V})\right.$, ad $\left.K\right)$. Let $\mathcal{Z}_{K}^{\bullet}(\mathcal{V})=\bigoplus_{k=-1}^{\infty} \mathcal{Z}_{K}^{k}$, where $\mathcal{Z}_{K}^{k}=$ $\operatorname{Ker}\left(\left.\operatorname{ad} K\right|_{W_{k}^{\text {var }}}\right)$, and $\mathcal{B}_{K}^{\bullet}(\mathcal{V})=\bigoplus_{k=-1}^{\infty} \mathcal{B}_{K}^{k}$, where $\mathcal{B}_{K}^{k}=(\operatorname{ad} K)\left(W_{k-1}^{\text {var }}\right)$. Then $\mathcal{Z}_{K}^{\bullet}(\mathcal{V})$ is a $\mathbb{Z}$-graded subalgebra of the Lie superalgebra $W^{\operatorname{var}}(\Pi \mathcal{V})$, and $\mathcal{B}_{K}^{\bullet}(\mathcal{V})$ is a $\mathbb{Z}$-graded ideal of $\mathcal{Z}_{K}^{\bullet}(\mathcal{V})$. Hence, the corresponding variational Poisson cohomology

$$
\mathcal{H}_{K}^{\bullet}(\mathcal{V})=\bigoplus_{k=-1}^{\infty} \mathcal{H}_{K}^{k}, \quad \mathcal{H}_{K}^{k}=\mathcal{Z}_{K}^{k} / \mathcal{B}_{K}^{k}
$$

is a $\mathbb{Z}$-graded Lie superalgebra.

In the special case when $K=\left(K_{i j}(\partial)\right)_{i, j \in I}$ has coefficients in $\mathcal{F}$, which, as in DSK11, we shall call a quasiconstant $\ell \times \ell$ matrix differential operator, formula (3.16) for the differential $\delta_{K}=$ ad $K$ becomes for $P \in W_{k-1}^{\mathrm{var}}, k \geq 0$, (3.19)

$$
\begin{aligned}
& \left(\delta_{K} P\right)_{i_{0}, \ldots, i_{k}}\left(\lambda_{0}, \ldots, \lambda_{k}\right) \\
& =(-1)^{k+1} \sum_{j \in I, n \in \mathbb{Z}_{+}} \sum_{\alpha=0}^{k}(-1)^{\alpha} \frac{\partial P_{i_{0}, \ldots, i_{k}}^{\alpha}\left(\lambda_{0}, \stackrel{\alpha}{\cdots}, \lambda_{k}\right)}{\partial u_{j}^{(n)}}\left(\lambda_{\alpha}+\partial\right)^{n} K_{j, i_{\alpha}}\left(\lambda_{\alpha}\right) .
\end{aligned}
$$

In fact, as shown in DSK11, Prop.9.9], if $K=\left(K_{i j}(\partial)\right)_{i, j \in I}$ is an arbitrary quasiconstant $\ell \times \ell$ matrix differential operator (not necessarily skewadjoint), then the same formula (3.19) still gives a well defined linear map $\delta_{K}: W_{k-1}^{\mathrm{var}} \rightarrow W_{k}^{\mathrm{var}}, k \geq 0$, such that $\delta_{K}^{2}=0$. Hence, we get a cohomology 
complex $\left(W^{\operatorname{var}}(\Pi \mathcal{V}), \delta_{K}\right)$. As before, we denote $\mathcal{Z}_{K}^{k}=\operatorname{Ker}\left(\left.\delta_{K}\right|_{W_{k}^{\text {var }}}\right), \mathcal{B}_{K}^{k}=$ $\delta_{K}\left(W_{k-1}^{\mathrm{var}}\right)$ and $\mathcal{H}_{K}^{k}=\mathcal{Z}_{K}^{k} / \mathcal{B}_{K}^{k}$.

For example, $\mathcal{H}_{K}^{-1}=\mathcal{Z}_{K}^{-1}=\left\{\int f \in \mathcal{V} / \partial \mathcal{V} \mid K^{*}(\partial) \frac{\delta f}{\delta u}=0\right\}$, which is called the set of central elements (or Casimir elements) of $K^{*}$. Next, we have (see DSK11):

$\mathcal{B}_{K}^{0}=\left\{K^{*}(\partial) \frac{\delta f}{\delta u}\right\}_{f \in \mathcal{V}}, \mathcal{Z}_{K}^{0}=\left\{P \in \mathcal{V}^{\ell} \mid D_{P}(\partial) \circ K(\partial)=K^{*}(\partial) \circ D_{P}^{*}(\partial)\right\}$.

Furthermore, given $P \in \mathcal{V}^{\ell}=W_{0}^{\text {var }}$, the element $\delta_{K} P \in W_{1}^{\text {var }}$, under the identification (3.11) of $W_{1}^{\mathrm{var}}$ with the space of $\ell \times \ell$ skewadjoint matrix differential operators, coincides with

$$
\delta_{K} P=D_{P}(\partial) \circ K(\partial)-K^{*}(\partial) \circ D_{P}^{*}(\partial) .
$$

Hence, $\mathcal{B}_{K}^{1}=\left\{D_{P}(\partial) \circ K(\partial)-K^{*}(\partial) \circ D_{P}^{*}(\partial)\right\}_{P \in \mathcal{V}^{\ell}}$. Finally, $\mathcal{Z}_{K}^{1}$ consists, under the same identification, of the $\ell \times \ell$ skewadjoint matrix differential operators $H(\partial)$ for which the RHS of (3.17) is zero.

Remark 3.4. If $\int f, \int g \in \mathcal{V} / \partial \mathcal{V}$, we have $\left[\int f, \int g\right]=0$ and

$$
\left[\delta_{K} \int f, \int g\right]-\left[\int f, \delta_{K} \int g\right]=\int\left(-\frac{\delta g}{\delta u} K^{*}(\partial) \frac{\delta f}{\delta u}-\frac{\delta f}{\delta u} K^{*}(\partial) \frac{\delta g}{\delta u}\right) .
$$

Hence, the differential $\delta_{K}$ in (3.19) is not an odd derivation unless $K(\partial)$ is skewadjoint. In particular, the corresponding cohomology $\mathcal{H}_{K}^{\bullet}(\mathcal{V})$ does not have a natural structure of a Lie superalgebra unless $K(\partial)$ is a skewadjoint operator.

3.4. The variational Poisson cohomology $H\left(W^{\operatorname{var}}(\Pi \mathcal{V}), \delta_{K}\right)$ for a quasiconstant matrix differential operator $K(\partial)$. Let $\mathcal{V}$ be an algebra of differential functions extension of $R_{\ell}$, the algebra of differential polynomials in the differential variables $u_{1}, \ldots, u_{\ell}$ over a differential field $\mathcal{F}$. Let $K=\left(K_{i j}(\partial)\right)_{i, j \in I}$ be a quasiconstant $\ell \times \ell$ matrix differential operator of order $N$ (not necessarily skewadjoint). For $k \geq-1$, we denote by $\mathcal{A}_{K}^{k} \subset W_{k}^{\text {var }}$ the subset consisting of arrays of the form

$$
\left(\sum_{j \in I}\left[P_{j, i_{0}, \ldots, i_{k}}\left(\lambda_{0}, \ldots, \lambda_{k}\right) u_{j}\right]\right)_{i_{0}, \ldots, i_{k} \in I},
$$

where $[x]$ denotes the coset of $x \in \mathcal{V}\left[\lambda_{0}, \ldots, \lambda_{k}\right]$ modulo $\left(\lambda_{0}+\cdots+\lambda_{k}+\right.$ d) $\mathcal{V}\left[\lambda_{0}, \ldots, \lambda_{k}\right]$, satisfying the following properties. For $j, i_{0}, \ldots, i_{k} \in I$, $P_{j, i_{0}, \ldots, i_{k}}\left(\lambda_{0}, \ldots, \lambda_{k}\right)$ are polynomials in $\lambda_{0}, \ldots, \lambda_{k}$ with coefficients in $\mathcal{F}$ of degree at most $N-1$ in each variable $\lambda_{i}$, skewsymmetric with respect to simultaneous permutations of the indices $i_{0}, \ldots, i_{k}$, and the variables $\lambda_{0}, \ldots, \lambda_{k}$, and satisfying the following condition:

$$
\begin{array}{r}
\sum_{\alpha=0}^{k+1}(-1)^{\alpha} \sum_{j \in I} P_{j, i_{0}, \stackrel{\alpha}{\cdots}, i_{k+1}}\left(\lambda_{0}, \stackrel{\alpha}{\ldots}, \lambda_{k+1}\right) K_{j i_{\alpha}}\left(\lambda_{\alpha}\right) \equiv 0 \\
\bmod \left(\lambda_{0}+\cdots+\lambda_{k+1}+\partial\right) \mathcal{F}\left[\lambda_{0}, \ldots, \lambda_{k+1}\right] .
\end{array}
$$


For example, $\mathcal{A}_{K}^{-1}$ consists of elements of the form $\sum_{j \in I} \int P_{j} u_{j} \in \mathcal{V} / \partial \mathcal{V}$, where $P \in \mathcal{F}^{\ell}$ solves the equation

$$
K^{*}(\partial) P=0 .
$$

In fact it is not hard to show that $\mathcal{A}_{K}^{-1}$ coincides with the set $\mathcal{Z}_{K}^{-1}$ of central elements of $K^{*}$ (see Lemma 4.4 below).

Next, $\mathcal{A}_{K}^{0}$ consists of elements of the form $\left(\sum_{j \in I} P_{i j}^{*}(\partial) u_{j}\right)_{i \in I} \in \mathcal{V}^{\ell}=$ $W_{0}^{\text {var }}$, where $P=\left(P_{i j}(\partial)\right)_{i, j \in I}$ is a quasiconstant $\ell \times \ell$ matrix differential operator of order at most $N-1$, solving the following equation:

$$
K^{*}(\partial) \circ P(\partial)=P^{*}(\partial) \circ K(\partial) \text {. }
$$

The description of the set $\mathcal{A}_{K}^{1}$ is more complicated. Given a polynomial in two variables $P(\lambda, \mu)=\sum_{m, n=0}^{N} c_{m n} \lambda^{m} \mu^{n} \in \mathcal{F}[\lambda, \mu]$, we denote $P^{* 1}(\lambda, \mu)=$ $\sum_{m, n=0}^{N}(-\lambda-\partial)^{m} c_{m n} \mu^{n}$, and $P^{* 2}(\lambda, \mu)=\sum_{m, n=0}^{N}(-\mu-\partial)^{n} c_{m n} \lambda^{m}$. Then, under the identification of $W_{1}^{\mathrm{var}}$ with the space of skewadjoint $\ell \times \ell$ matrix differential operators given by (3.11), $\mathcal{A}_{K}^{1}$ consists of operators $H=$ $\left(H_{i j}(\partial)\right)_{i, j \in I}$ of the form

$$
H_{i j}(\lambda)=-\sum_{k \in I} P_{k i j}^{*}(\lambda+\partial, \lambda) u_{k}
$$

where, for $i, j, k \in I, P_{k i j}(\lambda, \mu) \in \mathcal{F}[\lambda, \mu]$ are polynomials of degree at most $N-1$ in each variable, such that $P_{k i j}(\lambda, \mu)=-P_{k j i}(\mu, \lambda)$, and such that

$$
\begin{gathered}
\sum_{h \in I}\left(K_{i h}^{*}(\lambda+\mu+\partial) P_{h j k}(\lambda, \mu)+P_{h k i}^{* 2}(\mu, \lambda+\mu+\partial) K_{h j}(\lambda)\right. \\
\left.+P_{h i j}^{* 1}(\lambda+\mu+\partial, \lambda) K_{h k}(\mu)\right)=0 .
\end{gathered}
$$

Theorem 11.9 from [DSK11] can be stated as follows:

Theorem 3.5. Let $\mathcal{V}$ be a normal algebra of differential functions in $\ell$ differential variables over a linearly closed differential field $\mathcal{F}$, and let $\mathcal{C} \subset \mathcal{F}$ be the subfield of constants. Let $K(\partial)$ be a quasiconstant $\ell \times \ell$ matrix differential operator of order $N$ with invertible leading coefficient $K_{N} \in \operatorname{Mat}_{\ell \times \ell}(\mathcal{F})$. Then we have the following decomposition of $\mathcal{Z}_{K}^{k}$ in a direct sum of vector spaces over $\mathcal{C}$ :

$$
\mathcal{Z}_{K}^{k}=\mathcal{A}_{K}^{k} \oplus \mathcal{B}_{K}^{k}
$$

Hence, we have a canonical isomorphism $\mathcal{H}_{K}^{k} \simeq \mathcal{A}_{K}^{k}$. Moreover, $\mathcal{A}_{K}^{k}$ (hence $\left.\mathcal{H}_{K}^{k}\right)$ is a vector space over $\mathcal{C}$ of dimension $\left(\begin{array}{c}N \ell \\ k+2\end{array}\right)$.

Recall that, if $K$ is a skewadjoint operator, then $\mathcal{H}_{K}^{\bullet}(\mathcal{V})=\bigoplus_{k \geq-1} \mathcal{H}_{K}^{k}$ is a Lie superalgebra with consistent $\mathbb{Z}$-grading. In Section 5 we will prove the following

Theorem 3.6. Let $\mathcal{V}$ be a normal algebra of differential functions, over a linearly closed differential field $\mathcal{F}$. Let $K(\partial)$ be a quasiconstant skewadjoint $\ell \times \ell$ matrix differential operator of order $N$ with invertible leading coefficient 
$K_{N} \in \operatorname{Mat}_{\ell \times \ell}(\mathcal{F})$. Then the $\mathbb{Z}$-graded Lie superalgebra $\mathcal{H}_{K}^{\bullet}(\mathcal{V})$ is isomorphic to the $\mathbb{Z}$-graded Lie superalgebra $\widetilde{H}(N \ell, S)$ constructed in Section 2.2, where $S$ is the matrix, in some basis, of the nondegenerate symmetric bilinear form $\langle\cdot \mid \cdot\rangle_{K}^{0}$ constructed in Section 5.1 .

Remark 3.7. The subspace $\mathcal{A}_{K}^{\bullet}(\mathcal{V})=\bigoplus_{k=-1}^{\infty} \mathcal{A}_{K}^{k}$ is NOT, in general, a subalgebra of the Lie superalgebra $\mathcal{Z}^{\bullet}(\mathcal{V})$. We can enlarge it to be a subalgebra by letting $\widetilde{\mathcal{A}}_{K}^{k} \subset \mathcal{Z}_{K}^{k}$ be the subset consisting of arrays of the form (3.21) where $P_{j, i_{0}, \ldots, i_{k}}\left(\lambda_{0}, \ldots, \lambda_{k}\right)$ are polynomials in $\lambda_{0}, \ldots, \lambda_{k}$ with coefficients in $\mathcal{F}$ of arbitrary degree, skewsymmetric with respect to simultaneous permutations of the indices $i_{0}, \ldots, i_{k}$, and the variables $\lambda_{0}, \ldots, \lambda_{k}$, and satisfying condition (3.22). Then, clearly, $\mathcal{A}_{K}^{\bullet}(\mathcal{V}) \simeq \widetilde{\mathcal{A}}_{K}^{\bullet}(\mathcal{V}) /\left(\widetilde{\mathcal{A}}_{K}^{\bullet}(\mathcal{V}) \cap \mathcal{B}_{K}^{\bullet}(\mathcal{V})\right)$. For example, it is not hard to show that

$$
\widetilde{\mathcal{A}}_{K}^{0} \cap \mathcal{B}_{K}^{0}=\left\{S(\partial) K(\partial) \mid S^{*}(\partial)=S(\partial)\right\},
$$

so that, $\mathcal{A}_{K}^{0}$ is a Lie algebra, $\left\{S(\partial) K(\partial) \mid S^{*}(\partial)=S(\partial)\right\}$ is its ideal, and, by Theorem 3.6, the quotient is isomorphic to the Lie algebra $s o(N \ell)$.

Remark 3.8. If $N \leq 1$, then $\mathcal{A}_{K}^{\bullet}(\mathcal{V})$ is a subalgebra of the Lie superalgebra $\mathcal{Z}_{K}^{\bullet}(\mathcal{V})$, i.e. in this case the complex $\left(W^{\text {var }}(\Pi \mathcal{V})\right.$, ad $\left.K\right)$ is formal (cf. Get02]). However, this is not the case for $N>1$.

\section{Essential Variational Poisson COHOMOlogy}

In this section we introduce the subalgebra of essential variational Poisson cohomology and we prove a vanishing theorem for this cohomology.

4.1. The Casimir subalgebra $\mathcal{Z}_{K}^{-1} \subset \mathcal{V} / \partial \mathcal{V}$ and the essential subcomplex $\mathcal{E} W^{\operatorname{var}}(\Pi \mathcal{V})$. Throughout this section we let $\mathcal{V}$ be an algebra of differential functions in the variables $u_{i}, i \in I$, and we denote, as usual, by $\mathcal{F}$ the subalgebra of quasiconstant, and by $\mathcal{C} \subset \mathcal{F}$ the subalgebra of constants. Let $K=\left(K_{i j}(\partial)\right)_{i, j \in I}$ be a Hamiltonian $\ell \times \ell$ matrix differential operator with coefficients in $\mathcal{V}$. In other words, we can view $K$ as an element of $W_{1}^{\text {var }}$ such that $[K, K]=0$, hence, we can consider the corresponding cohomology complex $\left(W^{\operatorname{var}}(\Pi \mathcal{V})=\bigoplus_{k \geq-1} W_{k}^{\text {var }}\right.$, ad $\left.K\right)$. Recall from Section 3.3 that we have the $\mathbb{Z}$-graded subalgebra $\mathcal{Z}_{K}^{\bullet}(\mathcal{V})=\bigoplus_{k \geq-1} \mathcal{Z}_{K}^{k}$ of closed elements in $W^{\operatorname{var}}(\Pi \mathcal{V})$, and, inside it, the ideal of exact elements $\mathcal{B}^{\bullet}(\mathcal{V})=\bigoplus_{k \geq-1} \mathcal{B}_{K}^{k}$. The space $\mathcal{Z}_{K}^{-1}$ of central elements is, in this case,

$$
\mathcal{Z}_{K}^{-1}=\left\{C \in \mathcal{V} / \partial \mathcal{V} \mid[K, C]\left(=K(\partial) \frac{\delta C}{\delta u}\right)=0\right\} .
$$

We call an element $P \in W_{k}^{\mathrm{var}}$ essential if the following condition holds:

$$
\left[\ldots\left[\left[P, C_{0}\right], C_{1}\right], \ldots, C_{k}\right]=0, \forall C_{0}, \ldots, C_{k} \in \mathcal{Z}_{K}^{-1} .
$$

We denote by $\mathcal{E} W_{k}^{\text {var }} \subset W_{k}^{\text {var }}$ the subspace of essential elements. For example, $\mathcal{E} W_{-1}^{\mathrm{var}}=0$ and $\mathcal{E} W_{0}^{\mathrm{var}}$ consists of elements $P \in \mathcal{V}^{\ell}$ such that $\int P \frac{\delta C}{\delta u}=0$ for all central elements $C \in \mathcal{Z}_{K}^{-1}$. Furthermore, $\mathcal{E} W_{1}^{\text {var }}$ consists, under the 
identification (3.11), of skewadjoint $\ell \times \ell$ matrix differential operators $H(\partial)$, such that

$$
\int \frac{\delta C_{1}}{\delta u} H(\partial) \frac{\delta C_{2}}{\delta u}=0, \forall C_{1}, C_{2} \in \mathcal{Z}_{K}^{-1} .
$$

Let $\mathcal{E} W^{\mathrm{var}}=\bigoplus_{k \geq-1} \mathcal{E} W_{k}^{\mathrm{var}}$. This is a $\mathbb{Z}$-graded subspace of $W^{\mathrm{var}}(\Pi \mathcal{V})$, depending on the operator $K(\partial)$. Finally, denote by $\mathcal{E Z}_{K}^{\bullet}(\mathcal{V})=\bigoplus_{k>-1} \mathcal{E Z}_{K}^{k}$ the $\mathbb{Z}$-graded subspace of essentially closed elements, i.e. $\mathcal{E Z}_{K}^{k}=\mathcal{Z}_{K}^{k} \cap$ $\mathcal{E} W_{k}^{\mathrm{var}}$.

Proposition 4.1. (a) $\mathcal{E} W^{\mathrm{var}}$ is a $\mathbb{Z}$-graded subalgebra of the Lie superalgebra $W^{\operatorname{var}}(\Pi \mathcal{V})$. Consequently $\mathcal{E} \mathcal{Z}_{K}^{\bullet}(\mathcal{V})$ is a $\mathbb{Z}$-graded subalgebra of $\mathcal{E} W^{\text {var }}$.

(b) Exact elements are essentially closed, i.e. $B_{K}^{\bullet}(\mathcal{V}) \subset \mathcal{E Z}_{K}^{\bullet}(\mathcal{V})$, hence they form a $\mathbb{Z}$-graded ideal of the Lie superalgebra $\mathcal{E Z}_{K}^{\bullet}(\mathcal{V})$.

Proof. Let $P \in \mathcal{E} W_{h}^{\mathrm{var}}$ and $Q \in \mathcal{E} W_{k-h}^{\mathrm{var}}$, with $0 \leq h \leq k$, and let $C_{0}, \ldots, C_{k} \in$ $\mathcal{Z}_{K}^{-1}$. Using iteratively the Jacobi identity, we can express

$$
\left[\ldots\left[\left[[P, Q], C_{0}\right], C_{1}\right], \ldots, C_{k}\right]
$$

as a linear combination of the commutators of the pairs of elements of the form

$$
\left[\ldots\left[\left[P, C_{i_{0}}\right], C_{i_{1}}\right], \ldots, C_{i_{s-1}}\right] \text { and }\left[\ldots\left[\left[Q, C_{i_{s}}\right], C_{i_{s+1}}\right], \ldots, C_{i_{k}}\right]
$$

where $s$ is either $h$ or $h+1$. In the latter case the first element is zero since $P$ is essential, while in the former case the second element is zero since $Q$ is essential. Hence, $[P, Q]$ is essential. The second claim of part (a) follows since $\mathcal{E} \mathcal{Z}_{K}^{\bullet}(\mathcal{V})$ is the intersection of $\mathcal{E} W^{\text {var }}$ and $\mathcal{Z}_{K}^{\bullet}(\mathcal{V})$, which are both $\mathbb{Z}$-graded subalgebra of $W^{\operatorname{var}}(\Pi \mathcal{V})$.

For part (b), given the exact element $[K, P]$, where $P \in \mathcal{E} W_{k-1}^{\mathrm{var}}$, and given $C_{0}, \cdots, C_{k} \in \mathcal{Z}_{K}^{-1}$, we have, using again the Jacobi identity,

$$
\left[\ldots\left[\left[[K, P], C_{0}\right], C_{1}\right], \ldots, C_{k}\right]=\left[K,\left[\ldots\left[\left[P, C_{0}\right], C_{1}\right], \ldots, C_{k}\right]\right]=0 .
$$

So, we define the essential variational Poisson cohomology as

$$
\mathcal{E H}_{K}^{\bullet}(\mathcal{V})=\bigoplus_{k \geq-1} \mathcal{E} \mathcal{H}_{K}^{k} \text {, where } \mathcal{E} \mathcal{H}_{K}^{k}=\mathcal{E} \mathcal{Z}_{K}^{k} / \mathcal{B}_{K}^{k}
$$

Clearly, this is a $\mathbb{Z}$-graded subalgebra of the Lie superalgebra $\mathcal{H}_{K}^{\bullet}(\mathcal{V})=$ $H\left(W^{\operatorname{var}}(\Pi \mathcal{V}), \operatorname{ad} K\right)$.

Remark 4.2. Let $H(\partial)$ be a Hamiltonian operator compatible with $K(\partial)$, i.e. $[K, H]=0$. Suppose that the first step of the Lenard-Magri scheme always works, namely for every central element $C \in \mathcal{Z}_{K}^{-1}$ there exists $\int h \in$ $\mathcal{V} / \partial \mathcal{V}$ such that $[H, C]=\left[K, \int h\right]$. Then $H$ is essentially closed. Indeed, $\left[[H, C], C_{1}\right]=\left[\left[K, \int h\right], C_{1}\right]=\left[\int h,\left[K, C_{1}\right]\right]=0$ for every $C, C_{1} \in \mathcal{Z}_{K}^{-1}$. This is one of the reasons for the name "essential", since only for the essentially 
closed operators $H$ the Lenard-Magri scheme may work. Conversely, suppose $H(\partial)$ is an essentially closed Hamiltonian operator, i.e. $H(\partial) \in \mathcal{E Z}_{K}^{1}$. Then, for every central element $C \in \mathcal{Z}_{K}^{-1}$, it is immediate to see that there exists $\int h \in \mathcal{V} / \partial \mathcal{V}$ and $A \in \mathcal{E Z}_{K}^{0}$ such that $[H, C]=\left[K, \int h\right]+A$. If the first essential variational Poisson cohomology is zero, we can choose $A$ to be zero, which means that the first step in the Lenard-Magri scheme works.

4.2. Vanishing of the essential variational Poisson cohomology. In this section we prove the following

Theorem 4.3. If $\mathcal{V}$ be a normal algebra of differential functions in $\ell$ differential variables over a linearly closed differential field $\mathcal{F}$, and if $K(\partial)$ is a quasiconstant $\ell \times \ell$ matrix differential operator of order $N$ with invertible leading coefficient $K_{N} \in \operatorname{Mat}_{\ell \times \ell}(\mathcal{F})$, then $\mathcal{E H}_{K}^{\bullet}(\mathcal{V})=0$.

In order to prove Theorem 4.3 we will need some preliminary lemmas.

Lemma 4.4. Let $\mathcal{V}$ be an arbitrary algebra of differential functions. Let $K(\partial): \mathcal{V}^{\ell} \rightarrow \mathcal{V}^{\ell}$ be a quasiconstant $\ell \times \ell$ matrix differential operator with invertible leading coefficient $K_{N} \in \operatorname{Mat}_{\ell \times \ell}(\mathcal{F})$ Then:

(a) $\operatorname{Ker}(K(\partial))=\operatorname{Ker}\left(\left.K(\partial)\right|_{\mathcal{F}^{\ell}}\right)$.

(b) The map $\frac{\delta}{\delta u}: \mathcal{V} / \partial \mathcal{V} \rightarrow \mathcal{V}^{\ell}$ restricts to a surjective map $\frac{\delta}{\delta u}: \mathcal{Z}_{K}^{-1} \rightarrow$ $\operatorname{Ker}\left(\left.K(\partial)\right|_{\mathcal{F} \ell}\right)$.

(c) If, moreover, $\mathcal{V}$ is a normal algebra of differential functions and $\partial: \mathcal{F} \rightarrow$ $\mathcal{F}$ is surjective, then we have a bijection $\frac{\delta}{\delta u}: \mathcal{Z}_{K}^{-1} \stackrel{\sim}{\longrightarrow} \operatorname{Ker}\left(\left.K(\partial)\right|_{\mathcal{F} \ell}\right)$.

Proof. For part (a), we need to show that, if $F \in \mathcal{V}^{\ell}$ solves $K(\partial) F=0$, then $F \in \mathcal{F}^{\ell}$. Suppose, by contradiction, that $F \notin \mathcal{F}^{\ell}$. We may assume, without loss of generality, that $K_{N}=\mathbb{I}$, and that the first coordinate $F_{1}$ has maximal differential order, i.e. $F_{1}, \ldots, F_{\ell} \in \mathcal{V}_{n, i}$ and $F_{1} \notin \mathcal{V}_{n, i-1}$, for some $i \in I, n \in$ $\mathbb{Z}_{+}$. Then $\frac{\partial}{\partial u_{i}^{(n+N)}}(K(\partial) F)_{1}=\frac{\partial F_{1}}{\partial u_{i}^{(n)}} \neq 0$, a contradiction. Next, we prove part (b). The inclusion $\frac{\delta}{\delta u}\left(\mathcal{Z}_{K}^{-1}\right) \subset \operatorname{Ker}\left(\left.K(\partial)\right|_{\mathcal{F} \ell}\right)$ immediately follows from part (a). Furthermore, if $P \in \operatorname{Ker}\left(\left.K(\partial)\right|_{\mathcal{F} \ell}\right)$, then $C=\int \sum_{i} P_{i} u_{i} \in \mathcal{Z}_{K}^{-1}$ is such that $\frac{\delta C}{\delta u}=P$. Hence, $\frac{\delta}{\delta u}\left(\mathcal{Z}_{K}^{-1}\right)=\operatorname{Ker}\left(\left.K(\partial)\right|_{\mathcal{F}^{\ell}}\right)$, as desired. Finally, for part (c), if $\mathcal{V}$ is normal, we have by [BDSK09, Prop.1.5] that $\operatorname{Ker}\left(\frac{\delta}{\delta u}\right.$ : $\left.\mathcal{V} / \partial \mathcal{V} \rightarrow \mathcal{V}^{\ell}\right)=\mathcal{F} / \partial \mathcal{F}$, hence, if $\partial \mathcal{F}=\mathcal{F}$, we conclude that $\frac{\delta}{\delta u}: \mathcal{V} / \partial \mathcal{V} \rightarrow \mathcal{V}^{\ell}$ is injective.

To simplify notation, let $\mathcal{Z}:=\operatorname{Ker}(K(\partial))$. Under the assumptions of Theorem 4.3, by part (a) in Lemma 4.4, we have $\mathcal{Z} \subset \mathcal{F}^{\ell}$, and by part (c) we have a bijection

$$
\frac{\delta}{\delta u}: \mathcal{Z}_{K}^{-1} \stackrel{\sim}{\longrightarrow} \mathcal{Z}
$$


the inverse map being

$$
\mathcal{Z} \ni F=\left(\begin{array}{c}
f_{1} \\
\vdots \\
f_{\ell}
\end{array}\right) \mapsto \sum_{i} \int f_{i} u_{i} \in \mathcal{Z}_{K}^{-1} .
$$

Lemma 4.5. If $F_{1}, \ldots, F_{N \ell}$ are elements of $\mathcal{F}^{\ell}$, linearly independent over $\mathcal{C}$, and satisfying a differential equation

$$
F^{(N)}=A_{0} F+A_{1} F^{\prime}+\cdots+A_{N-1} F^{(N-1)},
$$

for some $A_{0}, \ldots, A_{N-1} \in \operatorname{Mat}_{\ell \times \ell}(\mathcal{F})$, then the vectors

$$
G_{1}:=\left(\begin{array}{l}
F_{1} \\
F_{1}^{\prime} \\
\vdots \\
F_{1}^{(N-1)}
\end{array}\right), \ldots, G_{N \ell}:=\left(\begin{array}{l}
F_{N \ell} \\
F_{N \ell}^{\prime} \\
\vdots \\
F_{N \ell}^{(N-1)}
\end{array}\right) \in \mathcal{F}^{N \ell}
$$

are linearly independent over $\mathcal{F}$.

Proof. Suppose by contradiction that

$$
a_{1} G_{1}+a_{2} G_{2}+\cdots+a_{N \ell} G_{N \ell}=0,
$$

is a nontrivial relation of linear dependence over $\mathcal{F}$. We can assume, without loss of generality, that such relation has minimal number of nonzero coefficients $a_{1}, \ldots, a_{N \ell} \in \mathcal{F}$, and that $a_{1}=1$. Note that equation (4.6) can be equivalently rewritten as the following system of equations in $\mathcal{F}^{\ell}$ :

$$
\begin{aligned}
& a_{1} F_{1}+a_{2} F_{2}+\cdots+a_{N \ell} F_{N \ell}=0 \\
& a_{1} F_{1}^{\prime}+a_{2} F_{2}^{\prime}+\cdots+a_{N \ell} F_{N \ell}^{\prime}=0 \\
& \cdots \\
& a_{1} F_{1}^{(N-1)}+a_{2} F_{2}^{(N-1)}+\cdots+a_{N \ell} F_{N \ell}^{(N-1)}=0
\end{aligned}
$$

Applying $\partial$ to both sides of equation (4.6), we get

$$
a_{1} G_{1}^{\prime}+a_{2} G_{2}^{\prime}+\cdots+a_{N \ell} G_{N \ell}^{\prime}+a_{1}^{\prime} G_{1}+a_{2}^{\prime} G_{2}+\cdots+a_{N \ell}^{\prime} G_{N \ell}=0 .
$$

The vector $a_{1} G_{1}^{\prime}+a_{2} G_{2}^{\prime}+\cdots+a_{N \ell} G_{N \ell}^{\prime}$ is an element of $\mathcal{F}^{N \ell}$ whose first $\ell$ coordinates are $a_{1} F_{1}^{\prime}+a_{2} F_{2}^{\prime}+\cdots+a_{N \ell} F_{N \ell}^{\prime}$, which are zero by the second equation in (4.7), the second $\ell$ coordinates are $a_{1} F_{1}^{(2)}+a_{2} F_{2}^{(2)}+\cdots+a_{N \ell} F_{N \ell}^{(2)}$, which are zero by the third equation in (4.7), and so on, up to the last set of $\ell$ coordinates, which are, by the equation (4.4),

$$
\begin{aligned}
& a_{1} F_{1}^{(N)}+a_{2} F_{2}^{(N)}+\cdots+a_{N \ell} F_{N \ell}^{(N)} \\
& =A_{0}\left(a_{1} F_{1}+a_{2} F_{2}+\cdots+a_{N \ell} F_{N \ell}\right)+A_{1}\left(a_{1} F_{1}^{\prime}+a_{2} F_{2}^{\prime}+\cdots+a_{N \ell} F_{N \ell}^{\prime}\right)+ \\
& \cdots+A_{N-1}\left(a_{1} F_{1}^{(N-1)}+a_{2} F_{2}^{(N-1)}+\cdots+a_{N \ell} F_{N \ell}^{(N-1)}\right)
\end{aligned}
$$

which is zero again by the equations (4.7). Hence, equation (4.8) reduces to

$$
a_{1}^{\prime} G_{1}+a_{2}^{\prime} G_{2}+\cdots+a_{N \ell}^{\prime} G_{N \ell}=0,
$$


which, by the assumption that $a_{1}=1$ and the minimality assumption on the coefficients of linear dependence (4.6), implies that all coefficients $a_{1}, \ldots, a_{N \ell}$ are constant. This, by the first equation in (4.7), contradicts the assumption that $F_{1}, \ldots, F_{N \ell}$ are linearly independent over $\mathcal{C}$.

Lemma 4.6. If $P(\partial)$ is a quasiconstant $m \times \ell(m \geq 1)$ matrix differential operator of order at most $N-1$ such that $P(\partial) F=0$ for every $F \in \mathcal{Z}=$ $\operatorname{Ker}(K(\partial))$, then $P(\partial)=0$.

Proof. Recall from [DSK11, Cor.A.3.7] that, if $K(\partial)=K_{0}+K_{1} \partial+\cdots+$ $K_{N} \partial^{N}$, with $K_{i} \in \operatorname{Mat}_{\ell \times \ell}(\mathcal{F}), i=0, \ldots, N$ and $K_{N}$ invertible, then the set of solutions in $\mathcal{F}^{\ell}$ of the homogeneous system $K(\partial) F=0$ is a vector space over $\mathcal{C}$ of dimension $N \ell$. Let $F_{1}, \ldots, F_{N \ell} \in \mathcal{F}^{\ell}$ be a basis of this space. Note that the equation $K(\partial) F=0$ has the form (4.4) with $A_{i}=-K_{N}^{-1} K_{i}, i=$ $0, \ldots, N-1$. Hence, by Lemma 4.5, all the vectors $G_{1}, \ldots, G_{N \ell}$ in 4.5) are linearly independent over $\mathcal{F}$, i.e. the Wronskian matrix

$$
W=\left(\begin{array}{llll}
F_{1} & F_{2} & \ldots & F_{N \ell} \\
F_{1}^{\prime} & F_{2}^{\prime} & \ldots & F_{N \ell}^{\prime} \\
& & \ldots & \\
F_{1}^{(N-1)} & F_{2}^{(N-1)} & \ldots & F_{N \ell}^{(N-1)}
\end{array}\right)
$$

is nondegenerate. By assumption $P(\partial) F_{1}=\cdots=P(\partial) F_{N \ell}=0$. Hence, letting $P(\partial)=P_{0}+P_{1} \partial+\cdots+P_{N-1} \partial^{N-1}$, where $P_{i} \in \operatorname{Mat}_{m \times \ell}(\mathcal{F})$, we get

$$
\left(P_{0}, P_{1}, \ldots, P_{N-1}\right) W=0,
$$

which, by the nondegeneracy of $W$, implies that $P_{0}=, \cdots=P_{N-1}=0$.

Proof of Theorem 4.3. Let $Q \in \mathcal{A}_{K}^{k}$. Recalling Theorem 3.5 and Proposition 4.1(b), it suffices to show that, if $Q$ is essential, then it is zero. By the definition of $\mathcal{A}_{K}^{k}$, we have, in particular, that $Q$ is an array with entries

$$
\begin{aligned}
& Q_{i_{0}, \ldots, i_{k}}\left(\lambda_{0}, \ldots, \lambda_{k}\right)=\sum_{j \in I} P_{j, i_{0}, \ldots, i_{k}}\left(\lambda_{0}, \ldots, \lambda_{k}\right) u_{j} \\
& \in \mathcal{V}\left[\lambda_{0}, \ldots, \lambda_{k}\right] /\left(\partial+\lambda_{0}+\cdots+\lambda_{k}\right) \mathcal{V}\left[\lambda_{0}, \ldots, \lambda_{k}\right],
\end{aligned}
$$

for some polynomials $P_{j, i_{0}, \ldots, i_{k}}\left(\lambda_{0}, \ldots, \lambda_{k}\right) \in \mathcal{F}\left[\lambda_{0}, \ldots, \lambda_{k}\right]$ of degree at most $N-1$ in each variable $\lambda_{i}$. Recalling formula (3.12), we have, for arbitrary $C_{0}, \ldots, C_{k} \in \mathcal{V} / \partial \mathcal{V}$

$$
\left[\ldots\left[\left[Q, C_{0}\right], C_{1}\right], \ldots, C_{k}\right]=\sum_{j, i_{0}, \ldots, i_{k} \in I} \int u_{j} P_{j, i_{0}, \ldots, i_{k}}\left(\partial_{0}, \ldots, \partial_{k}\right) \frac{\delta C_{0}}{\delta u_{i_{0}}} \ldots \frac{\delta C_{k}}{\delta u_{i_{k}}}
$$

where $\partial_{s}$ means $\partial$ acting on $\frac{\delta C_{s}}{\delta u_{i_{s}}}$. Hence, if $Q$ is essential, (4.9) is zero for all $C_{0}, \ldots, C_{k} \in \mathcal{Z}_{K}^{-1}$. By Lemma 4.4, we thus have

$$
\sum_{j, i_{0}, \ldots, i_{k} \in I} \int u_{j} P_{j, i_{0}, \ldots, i_{k}}\left(\partial_{0}, \ldots, \partial_{k}\right) F_{0} \ldots F_{k}=0
$$


for all $F_{0}, \ldots, F_{k} \in \operatorname{Ker}\left(\left.K(\partial)\right|_{\mathcal{F} \ell}\right)$. Since all coefficients of the $P_{j, i_{0}, \ldots, i_{k}}$ 's and all entries of the $F_{i}$ 's are quasiconstant, the above equation is equivalent to

$$
\sum_{i_{0}, \ldots, i_{k} \in I} P_{j, i_{0}, \ldots, i_{k}}\left(\partial_{0}, \ldots, \partial_{k}\right) F_{0} \ldots F_{k}=0, \forall j \in I .
$$

Applying Lemma 4.6 iteratively to each factor, we conclude that the polynomials $P_{j, i_{0}, \ldots, i_{k}}\left(\lambda_{0}, \ldots, \lambda_{k}\right)$ are zero.

Remark 4.7. By Remark 4.2, from the point of view of applicability of the Lenard-Magri scheme for a bi-Hamiltonian pair $(H, K)$, we should consider only essentially closed Hamiltonian operators $H(\partial)$. Moreover, by Theorem 4.3. if $K(\partial)$ is a quasiconstant matrix differential operator with invertible leading coefficient, an essentially closed $H(\partial)$ must be exact, namely, recalling equation (3.20), it must have the form

$$
H(\partial)=D_{P}(\partial) \circ K(\partial)+K(\partial) \circ D_{P}^{*}(\partial),
$$

for some $P \in \mathcal{V}^{\ell}$, and two such $P$ 's differ by an element of the form $K(\partial) \frac{\delta f}{\delta u}$ for some $\int f \in \mathcal{V} / \partial \mathcal{V}$.

Corollary 4.8. Under the assumptions of Theorem 4.3, the $\mathbb{Z}$-graded Lie superalgebra $\mathcal{H}_{K}^{\bullet}(\mathcal{V})$ is transitive.

Proof. By Theorem 4.3, if $P \in \mathcal{H}_{K}^{k}$ is such that $\left[\ldots\left[\left[P, C_{0}\right], C_{1}\right], \ldots, C_{k}\right]=0$ for every $C_{0}, \ldots, C_{k} \in \mathcal{Z}_{K}^{-1}=\mathcal{H}_{K}^{-1}$, then $P=0$. This, by definition, means that $\mathcal{H}_{K}^{\bullet}(\mathcal{V})$ is transitive.

\section{Isomorphism of $\mathbb{Z}$-GRAded Lie Superalgebras $\mathcal{H}_{K}^{\bullet}(\mathcal{V}) \simeq \widetilde{H}(N \ell, S)$}

In this section we introduce an inner product $\langle\cdot \mid \cdot\rangle_{K}: \mathcal{F}^{\ell} \times \mathcal{F}^{\ell} \rightarrow \mathcal{F}$ associated to an $\ell \times \ell$ matrix differential operator $K=\left(K_{i j}(\partial)\right)_{i, j \in I}$, which is used to prove Theorem 3.6.

5.1. The inner product associated to $K$. Let $\mathcal{F}$ be a differential algebra with derivation $\partial$, and denote by $\mathcal{C}$ the subalgebra of constants. As usual, we denote by $\cdot$ the standard inner product on $\mathcal{F}^{\ell}$, i.e. $F \cdot G=\sum_{i \in I} F_{i} G_{i} \in \mathcal{V}$ for $F, G \in \mathcal{V}^{\ell}$, where, as before, $I=\{1, \ldots, \ell\}$.

Consider the algebra of polynomials in two variables $\mathcal{F}[\lambda, \mu]$. Clearly, the map $\lambda+\mu+\partial: \mathcal{F}[\lambda, \mu] \rightarrow \mathcal{F}[\lambda, \mu]$ is injective. Hence, given $P(\lambda, \mu) \in$ $(\lambda+\mu+\partial) \mathcal{F}[\lambda, \mu]$, there is a unique preimage of this map in $\mathcal{F}[\lambda, \mu]$, that we denote by $(\lambda+\mu+\partial)^{-1} P(\lambda, \mu) \in \mathcal{F}[\lambda, \mu]$.

Let now $K(\partial)=\left(K_{i j}(\partial)\right)_{i, j \in I}$ be an arbitrary $\ell \times \ell$ matrix differential operator over $\mathcal{F}$. We expand its matrix entries as

$$
K_{i j}(\lambda)=\sum_{n=0}^{N} K_{i j ; n} \lambda^{n}, \quad K_{i j ; n} \in \mathcal{F} .
$$


The adjoint operator is $K^{*}(\partial)$, with entries

$$
K_{i j}^{*}(\lambda)=K_{j i}(-\lambda-\partial)=\sum_{n=0}^{N}(-\lambda-\partial)^{n} K_{j i ; n} .
$$

It follows from the expansions (5.1) and (5.2) that, for every $i, j \in I$, the polynomial $K_{i j}(\mu)-K_{j i}^{*}(\lambda)$ lies in the image of $\lambda+\mu+\partial$, so that we can consider the polynomial

$$
(\lambda+\mu+\partial)^{-1}\left(K_{i j}(\mu)-K_{j i}^{*}(\lambda)\right) \in \mathcal{F}[\lambda, \mu] .
$$

Next, for a polynomial $P(\lambda, \mu)=\sum_{m, n=0}^{N} p_{m n} \lambda^{m} \mu^{n} \in \mathcal{F}[\lambda, \mu]$, we use the following notation

$$
P(\lambda, \mu)\left(\left.\right|_{\lambda=\partial} f\right)\left(\left.\right|_{\mu=\partial} g\right):=\sum_{m, n=0}^{N} p_{m n}\left(\partial^{m} f\right)\left(\partial^{n} g\right) \cdot\left(\left.{ }_{\lambda}\right|_{=\partial} f\right)
$$

Based on the observation (5.3), and using the notation in (5.4), we define the following inner product $\langle\cdot \mid \cdot\rangle_{K}: \mathcal{F}^{\ell} \times \mathcal{F}^{\ell} \rightarrow \mathcal{F}$, associated to $K=$ $\left(K_{i j}(\partial)\right)_{i, j \in I} \in \operatorname{Mat}_{\ell \times \ell}(\mathcal{F}[\partial])$ :

$$
\langle F \mid G\rangle_{K}=\sum_{i, j \in I}(\lambda+\mu+\partial)^{-1}\left(K_{i j}(\mu)-K_{j i}^{*}(\lambda)\right)\left(\left.\right|_{\lambda=\partial} F_{i}\right)\left(\left.\right|_{\mu=\partial} G_{j}\right) .
$$

It is not hard to write an explicit formula for $\langle F \mid G\rangle_{K}$, using the expansion (5.1) for $K_{i j}(\lambda)$ :

$$
\langle F \mid G\rangle_{K}=\sum_{i, j \in I} \sum_{n=0}^{N} \sum_{m=0}^{n-1}\left(\begin{array}{c}
n \\
m
\end{array}\right)(-\partial)^{n-1-m}\left(F_{i} K_{i j ; n} \partial^{m} G_{j}\right) .
$$

Lemma 5.1. For every $F, G \in \mathcal{V}^{\ell}$, we have

$$
\partial\langle F \mid G\rangle_{K}=F \cdot K(\partial) G-G \cdot K^{*}(\partial) F .
$$

Proof. It immediately follows from the definition (5.5) of $\langle F \mid G\rangle_{K}$.

Lemma 5.2. For every $K(\partial) \in \operatorname{Mat}_{\ell \times \ell}(\mathcal{F}[\partial])$ and $F, G \in \mathcal{F}^{\ell}$, we have

$$
\langle G \mid F\rangle_{K^{*}}=-\langle F \mid G\rangle_{K}
$$

In particular, the inner product $\langle\cdot \mid \cdot\rangle_{K}$ is symmetric (respectively skewsymmetric) if $K$ is skewadjoint (resp. selfadjoint).

Proof. By equation (5.5) we have

$$
\begin{aligned}
& \langle G \mid F\rangle_{K^{*}}=\sum_{i, j \in I}(\lambda+\mu+\partial)^{-1}\left(K_{i j}^{*}(\mu)-K_{j i}(\lambda)\right)\left(\left.\right|_{\lambda=\partial} G_{i}\right)\left(\left.\right|_{\mu=\partial} F_{j}\right) \\
& =-\sum_{i, j \in I}(\lambda+\mu+\partial)^{-1}\left(K_{i j}(\mu)-K_{j i}^{*}(\lambda)\right)\left(\left.\right|_{\lambda=\partial} F_{i}\right)\left(\left.\right|_{\mu=\partial} G_{j}\right)=-\langle F \mid G\rangle_{K} .
\end{aligned}
$$


Following the notation of the previous sections, we let $\mathcal{Z}=\operatorname{Ker}(K(\partial)) \subset$ $\mathcal{F}^{\ell}$. Clearly, $\mathcal{Z}$ is a submodule of the $\mathcal{C}$-module $\mathcal{F}^{\ell}$.

Lemma 5.3. If $K(\partial) \in \operatorname{Mat}_{\ell \times \ell}(\mathcal{F}[\partial])$ is skewadjoint, then $\langle F \mid G\rangle_{K} \in \mathcal{C}$ for every $F, G \in \mathcal{Z}$

Proof. It is an immediate consequence of Lemma 5.1.

According to Lemmas 5.2 and 5.3, if $K(\partial) \in \operatorname{Mat}_{\ell \times \ell}(\mathcal{F}[\partial])$ is skewadjoint, the restriction of $\langle\cdot \mid \cdot\rangle_{K}$ to $\mathcal{Z} \subset \mathcal{F}^{\ell}$ defines a symmetric bilinear form on $\mathcal{Z}$ with values in $\mathcal{C}$, which we denote by

$$
\langle\cdot \mid \cdot\rangle_{K}^{0}:=\left.\langle\cdot \mid \cdot\rangle_{K}\right|_{\mathcal{Z}}: \mathcal{Z} \times \mathcal{Z} \rightarrow \mathcal{C} .
$$

Lemma 5.4. Assuming that $K(\partial) \in$ Mat $_{\ell \times \ell}(\mathcal{F}[\partial])$ is a skewadjoint operator and $P(\partial) \in \operatorname{Mat}_{\ell \times \ell}(\mathcal{F}[\partial])$ is such that $K(\partial) P(\partial)+P^{*}(\partial) K(\partial)=0$, we have

$$
\langle P(\partial) F \mid G\rangle_{K}+\langle F \mid P(\partial) G\rangle_{K}=0
$$

for every $F, G \in \mathcal{F}^{\ell}$.

Proof. By equation (5.5), we have

$$
\begin{aligned}
& \langle P(\partial) F \mid G\rangle_{K} \\
& =\sum_{i, j, k \in I}(\lambda+\mu+\partial)^{-1}\left(K_{k j}(\mu)+K_{j k}(\lambda)\right)\left(\left.\right|_{\lambda=\partial} P_{k i}(\partial) F_{i}\right)\left(\left.\right|_{\mu=\partial} G_{j}\right) \\
& =\sum_{i, j, k \in I}(\lambda+\mu+\partial)^{-1}\left(K_{k j}(\mu)+K_{j k}(\lambda+\partial)\right) P_{k i}(\lambda)\left(\left.\right|_{\lambda=\partial} F_{i}\right)\left(\left.\right|_{\mu=\partial} G_{j}\right) \\
& =\sum_{i, j, k \in I}(\lambda+\mu+\partial)^{-1}\left(P_{k i}(\lambda) K_{k j}(\mu)-P_{j k}^{*}(\lambda+\mu) K_{k i}(\lambda)\right)\left(\left.\right|_{\lambda=\partial} F_{i}\right)\left(\left.\right|_{\mu=\partial} G_{j}\right) .
\end{aligned}
$$

In the last identity we used the assumption that $K(\partial) P(\partial)=-P^{*}(\partial) K(\partial)$. Similarly,

$$
\begin{aligned}
& \langle F \mid P(\partial) G\rangle_{K}=\sum_{i, j, k \in I}(\lambda+\mu+\partial)^{-1} \\
& \times\left(-P_{i k}^{*}(\mu+\partial) K_{k j}(\mu)+P_{k j}(\mu) K_{k i}(\lambda)\right)\left(\left.\right|_{\lambda=\partial} F_{i}\right)\left(\left.\right|_{\mu=\partial} G_{j}\right) .
\end{aligned}
$$

Combining these two equations, we get

$$
\begin{aligned}
& \langle P(\partial) F \mid G\rangle_{K}+\langle F \mid P(\partial) G\rangle_{K} \\
& =\sum_{i, j, k \in I}(\lambda+\mu+\partial)^{-1}\left(\left(P_{k i}(\lambda)-P_{i k}^{*}(\mu+\partial)\right) K_{k j}(\mu)\right. \\
& \left.\quad+\left(P_{k j}(\mu)-P_{j k}^{*}(\lambda+\mu)\right) K_{k i}(\lambda)\right)\left(\left.\right|_{\lambda=\partial} F_{i}\right)\left(\left.\right|_{\mu=\partial} G_{j}\right) .
\end{aligned}
$$

We next observe that the differential operator $P_{k i}(\lambda)-P_{i k}^{*}(\mu+\partial)$ lies in $(\lambda+\mu+\partial) \circ(\mathcal{F}[\lambda, \mu])[\partial]$, i.e. it is of the form

$$
P_{k i}(\lambda)-P_{i k}^{*}(\mu+\partial)=(\lambda+\mu+\partial) \circ Q_{k i}(\lambda, \mu+\partial),
$$

for some polynomial $Q_{k i}$. Hence,

$$
(\lambda+\mu+\partial)^{-1}\left(P_{k i}(\lambda)-P_{i k}^{*}(\mu+\partial)\right) K_{k j}(\mu)\left(\left.\right|_{\mu=\partial} G_{j}\right)=Q_{i k}(\lambda, \partial) K_{k j}(\partial) G_{j},
$$


which, after summing with respect to $j \in I$, becomes zero since, by assumption, $G \in \operatorname{Ker}(K(\partial))$. Similarly,

$$
(\lambda+\mu+\partial)^{-1}\left(P_{k j}(\mu)-P_{j k}^{*}(\lambda+\mu)\right) K_{k i}(\lambda)\left(\left.\right|_{\lambda=\partial} F_{i}\right)=Q_{k j}(\mu, \partial) K_{k i}(\partial) F_{i},
$$

which is zero after summing with respect to $i \in I$, since $F \in \operatorname{Ker}(K(\partial))$. Therefore the RHS of (5.7) is zero, proving the claim.

Proposition 5.5. Assuming that $\mathcal{F}$ is a linearly closed differential field, and that $K(\partial) \in \operatorname{Mat}_{\ell \times \ell}(\mathcal{F}[\partial])$ is a skewadjoint $\ell \times \ell$ matrix differential operator with invertible leading coefficient, the $\mathcal{C}$-bilieanr form $\langle\cdot \mid \cdot\rangle_{K}^{0}: \mathcal{Z} \times \mathcal{Z} \rightarrow \mathcal{C}$ is nondegenerate.

Proof. Given $F \in \mathcal{F}^{\ell}$, consider the map $P_{F}: \mathcal{F}^{\ell} \rightarrow \mathcal{F}$ given by $G \mapsto$ $P_{F}(G)=\langle F \mid G\rangle_{K}^{0}$. Equation (5.6) can be rewritten by saying that $P_{F}$ is a $1 \times \ell$ matrix differential operator, of order less than or equal to $N-1$, with entries

$$
\left(P_{F}\right)_{j}(\partial)=\sum_{i \in I} \sum_{n=0}^{N} \sum_{m=0}^{n-1}\left(\begin{array}{c}
n \\
m
\end{array}\right)(-\partial)^{n-1-m} \circ F_{i} K_{i j ; n} \partial^{m} .
$$

Suppose now that $P_{F}(G)=\langle P \mid G\rangle_{K}^{0}=0$ for all $G \in \mathcal{Z} \subset \mathcal{F}^{\ell}$. By Lemma 4.6 we get that $P_{F}(\partial)=0$. On the other hand, the (left) coefficient of $\partial^{N-1}$ in $\left(P_{F}\right)_{j}(\partial)$ is

$$
0=\sum_{i \in I} \sum_{m=0}^{N-1}\left(\begin{array}{l}
N \\
m
\end{array}\right)(-1)^{N-1-m} F_{i}\left(K_{N}\right)_{i j}=\sum_{i \in I} F_{i}\left(K_{N}\right)_{i j} .
$$

Since, by assumption, $K_{N} \in \operatorname{Mat}_{\ell \times \ell}(\mathcal{F})$ is invertible, we conclude that $F=$ 0 .

5.2. Proof of Theorem [3.6. Recall from Lemma 4.4 that $\mathcal{H}_{K}^{-1}=\mathcal{Z}_{K}^{-1}$ is isomorphic, as a $\mathcal{C}$-vector space, to $\mathcal{Z}=\operatorname{Ker}(K(\partial))$, and, from Theorem 3.5 , that $\operatorname{dim}_{\mathcal{C}} \mathcal{Z}=N \ell$. By Corollary 4.3 , the $\mathbb{Z}$-graded Lie superalgebra $\mathcal{H}_{K}^{\bullet}(\mathcal{V})$ is transitive, i.e. if $P \in \mathcal{H}_{K}^{k}, k \geq 0$, is such that $\left[P, \mathcal{H}_{K}^{-1}\right]=0$, then $P=0$. Hence, due to transitivity, the representation of $\mathcal{H}^{0}$ on $\mathcal{H}_{K}^{-1}=\mathcal{Z}_{K}^{-1}$ is faithful. Identifying $\mathcal{Z}_{K}^{-1} \simeq \mathcal{Z}$, we can therefore view $\mathcal{H}_{K}^{0}$ as a subalgebra of the Lie algebra $g l(\mathcal{Z})=g l_{N \ell}$. Recall, from Theorem 3.5 that $\mathcal{H}_{K}^{0} \simeq \mathcal{A}_{K}^{0}$ consists of elements of the form $Q=\left(\sum_{j} P_{i j}^{*}(\partial) u_{j}\right)_{i \in I} \in \mathcal{V}^{\ell}$, where $P(\partial)=\left(P_{i j}(\partial)\right)_{i \in I}$ is an $\ell \times \ell$ matrix differential operator of order at most $N-1$ solving equation (3.23). Moreover, by (3.13), the bracket of an element $Q \in \mathcal{H}_{K}^{0}$ as above and an element $C \in \mathcal{Z}_{K}^{-1}=\mathcal{H}_{K}^{-1} \subset \mathcal{V} / \partial \mathcal{V}$, is given by

$$
[Q, C]=\sum_{i, j \in I} \int\left(P_{i j}^{*}(\partial) u_{j}\right) \frac{\delta C}{\delta u_{i}}=\sum_{i, j \in I} \int u_{i} P_{i j}(\partial) \frac{\delta C}{\delta u_{j}} .
$$

Hence, by the identification (4.3), the corresponding action of $Q \in \mathcal{H}_{K}^{0}$ on $\mathcal{Z} \subset \mathcal{F}^{\ell}$ is simply given by the standard action of the $\ell \times \ell$ matrix differential operator $P(\partial)$ on $\mathcal{F}^{\ell}$. By Lemmas 5.2 and 5.3 and by Proposition $5.5,\langle\cdot \mid \cdot\rangle_{K}^{0}$ 
is a nondegenerate symmetric bilinear form on $\mathcal{Z}$, and by Lemma 5.4 it is invariant with respect to this action of $Q \in \mathcal{H}_{K}^{0}$ on $\mathcal{Z}$. Hence, the image of $\mathcal{H}_{K}^{0}$ via the above embedding $\mathcal{H}_{K}^{0} \rightarrow g l(\mathcal{Z})$, is a subalgebra of $s o\left(\mathcal{Z},\langle\cdot \mid \cdot\rangle_{K}^{0}\right)$. Due to transitivity of the $\mathbb{Z}$-graded Lie superalgebra $\mathcal{H}_{K}^{\bullet}(\mathcal{V})$, it embeds in the full prolongation of the pair $\left(\mathcal{Z}, s o\left(\mathcal{Z},\langle\cdot \mid \cdot\rangle_{K}^{0}\right)\right)$, which, by Proposition 2.2, is isomorphic to $\widetilde{H}(N \ell, S)$, where $S$ is the $N \ell \times N \ell$ matrix of the bilinear form $\langle\cdot \mid \cdot\rangle_{K}^{0}$, in some basis. By Theorem 3.5, $\operatorname{dim}_{\mathcal{C}} \mathcal{H}_{K}^{k}=\left(\begin{array}{c}N \ell \\ k+2\end{array}\right)$, which is equal

to $\operatorname{dim}_{\mathcal{C}} \widetilde{H}_{k}(N \ell, S)$. We thus conclude that the $\mathbb{Z}$-graded Lie superalgebras $\mathcal{H}_{K}^{\bullet}(\mathcal{V})$ and $\widetilde{H}(N \ell, S)$ are isomorphic.

Remark 5.6. The same arguments as above show that, without any assumption on the algebra of differential functions $\mathcal{V}$ and on the differential field $\mathcal{F}$ (with subfield of constants $\mathcal{C}$ ), and for every Hamiltonian operator $K$ (not necessarily quasiconstant nor with invertible leading coefficient), we have an injective homomorphism of $\mathbb{Z}$-graded Lie superalgebras $\mathcal{H}_{K}^{\bullet}(\mathcal{V}) / \mathcal{E} \mathcal{H}_{K}^{\bullet}(\mathcal{V}) \rightarrow W(n)$, where $n=\operatorname{dim}_{\mathcal{C}}\left(\mathcal{H}_{K}^{-1}\right)$.

\section{Translation invariant VARiational Poisson COHOMOlOGy}

In the previous sections we studied the variational Poisson cohomology $\widetilde{H}_{K}^{\bullet}(\mathcal{V})$ in the simplest case when the differential field of quasiconstants $\mathcal{F} \subset \mathcal{V}$ is linearly closed. In this section we consider the other extreme case, often studied in literature - the translation invariant case, when $\mathcal{F}=\mathcal{C}$.

6.1. Upper bound of the dimension of the translation invariant variational Poisson cohomology. Let $\mathcal{V}$ be a normal algebra of differential functions, and assume that it is translation invariant, i.e. the differential field $\mathcal{F}$ of quasiconstants coincides with the field $\mathcal{C}$ of constants. Let $K(\partial)$ be an $\ell \times \ell$ matrix differential operator of order $N$, with coefficients in Mat $_{\ell \times \ell}(\mathcal{C})$, and with invertible leading coefficient $K_{N}$.

For $k \geq-1$, denote by $\widetilde{\mathcal{H}}^{k}$ the space of arrays $\left(P_{i_{0}, \ldots, i_{k}}\left(\lambda_{0}, \ldots, \lambda_{k}\right)\right)_{i_{0}, \ldots, i_{k} \in I}$ with entries $P_{i_{0}, \ldots, i_{k}}\left(\lambda_{0}, \ldots, \lambda_{k}\right) \in \mathcal{C}\left[\lambda_{0}, \ldots, \lambda_{k}\right]$, of degree at most $N-1$ in each variable, which are skewsymmetric with respect to simultaneous permutations of the indices $i_{0}, \ldots, i_{k}$ and the variables $\lambda_{0}, \ldots, \lambda_{k}$ (in the notation of [DSK11], $\left.\widetilde{\mathcal{H}}^{k}=\widetilde{\Omega}_{0,0}^{k-1}\right)$. In particular, $\widetilde{\mathcal{H}}^{-1}=\mathcal{C}$. Note that, for $k \geq-1$, we have

$$
\operatorname{dim}_{\mathcal{C}} \widetilde{\mathcal{H}}^{k}=\left(\begin{array}{c}
N \ell \\
k+1
\end{array}\right)
$$

The long exact sequence [DSK11, eq.(11.4)] becomes (in the notation of the present paper):

$$
\begin{aligned}
& 0 \rightarrow \mathcal{C} \stackrel{\beta_{-1}}{\longrightarrow} \mathcal{H}_{K}^{-1} \stackrel{\gamma_{-1}}{\longrightarrow} \widetilde{\mathcal{H}}^{0} \stackrel{\alpha_{0}}{\longrightarrow} \widetilde{\mathcal{H}}^{0} \stackrel{\beta_{0}}{\longrightarrow} \ldots \\
& \ldots \stackrel{\gamma_{k-1}}{\longrightarrow} \widetilde{\mathcal{H}}^{k} \stackrel{\alpha_{k}}{\longrightarrow} \widetilde{\mathcal{H}}^{k} \stackrel{\beta_{k}}{\longrightarrow} \mathcal{H}_{K}^{k} \stackrel{\gamma_{k}}{\longrightarrow} \widetilde{\mathcal{H}}^{k+1} \stackrel{\alpha_{k+1}}{\longrightarrow} \widetilde{\mathcal{H}}^{k+1} \stackrel{\beta_{k+1}}{\longrightarrow} \ldots
\end{aligned}
$$


For every $k \geq-1$, we have $\operatorname{dim}_{\mathcal{C}}\left(\mathcal{H}_{K}^{k}\right)=\operatorname{dim}_{\mathcal{C}}\left(\operatorname{Ker} \gamma_{k}\right)+\operatorname{dim}_{\mathcal{C}}\left(\operatorname{Im} \gamma_{k}\right)$. By exactness of the sequence (6.2), we have that $\operatorname{dim}_{\mathcal{C}}\left(\operatorname{Im} \gamma_{k}\right)=\operatorname{dim}_{\mathcal{C}}\left(\operatorname{Ker} \alpha_{k+1}\right)$, and $\operatorname{dim}_{\mathcal{C}}\left(\operatorname{Ker} \gamma_{k}\right)=\operatorname{dim}_{\mathcal{C}}\left(\operatorname{Im} \beta_{k}\right)$. Moreover, $\operatorname{dim}_{\mathcal{C}}\left(\operatorname{Im} \beta_{-1}\right)=1$ and, for $k \geq 0$, we have, again by exactness of (6.2), that $\operatorname{dim}_{\mathcal{C}}\left(\operatorname{Im} \beta_{k}\right)=\operatorname{dim}_{\mathcal{C}} \widetilde{\mathcal{H}}^{k}-$ $\operatorname{dim}_{\mathcal{C}}\left(\operatorname{Ker} \beta_{k}\right)=\operatorname{dim}_{\mathcal{C}} \widetilde{\mathcal{H}}^{k}-\operatorname{dim}_{\mathcal{C}}\left(\operatorname{Im} \alpha_{k}\right)=\operatorname{dim}_{\mathcal{C}}\left(\operatorname{Ker} \alpha_{k}\right)$. Hence, using (6.1) we conclude that

$$
\operatorname{dim}_{\mathcal{C}}\left(\mathcal{H}_{K}^{-1}\right)=1+\operatorname{dim}_{\mathcal{C}}\left(\operatorname{Ker} \alpha_{0}\right) \leq N \ell+1,
$$

and, for $k \geq 0$ (by the Tartaglia-Pascal triangle),

$$
\operatorname{dim}_{\mathcal{C}}\left(\mathcal{H}_{K}^{k}\right)=\operatorname{dim}_{\mathcal{C}}\left(\operatorname{Ker} \alpha_{k}\right)+\operatorname{dim}_{\mathcal{C}}\left(\operatorname{Ker} \alpha_{k+1}\right) \leq\left(\begin{array}{c}
N \ell+1 \\
k+2
\end{array}\right) .
$$

Recalling equation (4.1), we have $\mathcal{H}_{K}^{-1}=\mathcal{Z}_{K}^{-1}=\left\{\int f \in \mathcal{V} / \partial \mathcal{V} \mid K(\partial) \frac{\delta f}{\delta u}=\right.$ $0\}$. By Lemma 4.4(b) we have a surjective map $\frac{\delta}{\delta u}: \mathcal{H}_{K}^{-1} \rightarrow \operatorname{Ker}\left(\left.K(\partial)\right|_{\mathcal{C}^{\ell}}\right)$. Recall that, if $\mathcal{V}$ is a normal algebra of differential functions, we have $\operatorname{Ker}\left(\frac{\delta}{\delta u}: \mathcal{V} \rightarrow \mathcal{V}^{\ell}\right)=\mathcal{C}+\partial \mathcal{V}$ [BDSK09]. It follows that $\operatorname{Ker}\left(\left.\frac{\delta}{\delta u}\right|_{\mathcal{H}_{K}^{-1}}\right)=$ $\operatorname{Ker}\left(\left.\frac{\delta}{\delta u}\right|_{\mathcal{V} / \partial \mathcal{V}}\right) \simeq \mathcal{C}$. Therefore,

$$
\mathcal{H}_{K}^{-1}=\int \mathcal{C} \oplus\left\{\int u A \mid A \in \operatorname{Ker}\left(K_{0}\right) \subset \mathcal{C}^{\ell}\right\},
$$

where, $u=\left(u_{1}, \ldots, u_{\ell}\right)$, and $K_{0}=K(0)$ is the constant coefficient of the differential operator $K(\partial)$. Hence,

$$
\operatorname{dim}_{\mathcal{C}}\left(\mathcal{H}_{K}^{-1}\right)=1+\operatorname{dim}_{\mathcal{C}}\left(\operatorname{Ker} K_{0}\right)=1+\ell-\operatorname{rk}\left(K_{0}\right) .
$$

In conclusion, the inequality in (6.3) is a strict inequality unless $K(\partial)$ has order 1 with $K_{0}=0$, i.e. $K(\partial)=S \partial$, where $S \in$ Mat $_{\ell \times \ell}(\mathcal{C})$ is a nondegenerate matrix.

Remark 6.1. The map $\alpha_{k}: \widetilde{\mathcal{H}}^{k} \rightarrow \widetilde{\mathcal{H}}^{k}$ can be constructed as follows [DSK11. Let $P=\left(P_{i_{0}, \ldots, i_{k}}\left(\lambda_{0}, \ldots, \lambda_{k}\right)\right)_{i_{0}, \ldots, i_{k} \in I}$ be in $\widetilde{\mathcal{H}}^{k}$, i.e. $P_{i_{0}, \ldots, i_{k}}\left(\lambda_{0}, \ldots, \lambda_{k}\right)$ are polynomials of degree at most $N-1$ in each variable $\lambda_{i}$ with coefficients in $\mathcal{C}$, skewsymmetric with respect to simultaneous permutations in the indices $i_{0}, \ldots, i_{k}$ and the variables $\lambda_{0}, \ldots, \lambda_{k}$. Then, there exist a unique element $\alpha_{k}(P):=R=\left(R_{i_{0}, \ldots, i_{k}}\left(\lambda_{0}, \ldots, \lambda_{k}\right)\right)_{i_{0}, \ldots, i_{k} \in I} \in \widetilde{\mathcal{H}}^{k}$ and a (unique) array $Q=\left(Q_{j, i_{1}, \ldots, i_{k}}\left(\lambda_{1}, \ldots, \lambda_{k}\right)\right)_{j, i_{1}, \ldots, i_{k} \in I}$, where $Q_{j, i_{1}, \ldots, i_{k}}\left(\lambda_{1}, \ldots, \lambda_{k}\right)$ are polynomials of degree at most $N-1$ in each variable, with coefficients in $\mathcal{C}$, skewsymmetric with respect of simultaneous permutations of the indices $i_{1}, \ldots, i_{k}$ and the variables $\lambda_{1}, \ldots, \lambda_{k}$, such that the following identity holds in $\mathcal{C}\left[\lambda_{0}, \ldots, \lambda_{k}\right]$ :

$$
\begin{gathered}
\left(\lambda_{0}+\cdots+\lambda_{k}\right) P_{i_{0}, \ldots, i_{k}}\left(\lambda_{0}, \ldots, \lambda_{k}\right)=R_{i_{0}, \ldots, i_{k}}\left(\lambda_{0}, \ldots, \lambda_{k}\right) \\
+\sum_{\alpha=0}^{k}(-1)^{\alpha} \sum_{j \in I} Q_{j, i_{0}, \ldots, i_{k}}^{\alpha}\left(\lambda_{0}, \stackrel{\alpha}{\alpha}, \lambda_{k}\right) K_{j i_{\alpha}}\left(\lambda_{\alpha}\right) .
\end{gathered}
$$


Hence, $\operatorname{Ker}\left(\alpha_{k}\right)$ is in bijection with the space $\Sigma_{k}$ of arrays $Q$ as above, satisfying the condition:

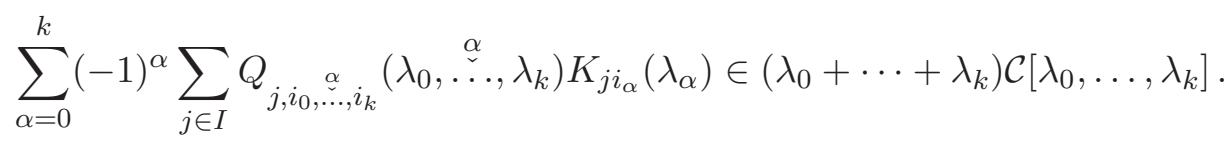

For example, $\Sigma_{0}=\left\{Q \in \mathcal{C}^{\ell} \mid K_{0}^{T} Q=0\right\}$, hence its dimension equals $\operatorname{dim}_{\mathcal{C}}\left(\operatorname{Ker} \alpha_{0}\right)=\operatorname{dim}\left(\operatorname{Ker} K_{0}\right)=\ell-\operatorname{rk}\left(K_{0}\right)$ (in accordance with (6.5)). Furthermore, $\Sigma_{1}$ consists of polynomials $Q(\lambda)$ with coefficients in $\operatorname{Mat}_{\ell \times \ell}(\mathcal{C})$, of degree at most $N-1$, such that

$$
K^{T}(-\lambda) Q(\lambda)=Q^{T}(-\lambda) K(\lambda) .
$$

Remark 6.2. It is clear from Remark 6.1 that, while in the linearly closed case, the Lie superalgebra $\mathcal{H}_{K}^{\bullet}(\mathcal{V})$ depends only on $\ell$ and the order $N$ of $K(\partial)$, in the translation invariant case $\mathcal{F}=\mathcal{C}$ the dimension of $\mathcal{H}_{K}^{\bullet}(\mathcal{V})$ depends essentially on the operator $K(\partial)$. Hence, in this sense, the choice of an algebra $\mathcal{V}$ over a linearly closed differential field $\mathcal{F}$ seems to be a more natural one. This is the key message of the paper.

In the next section we study in more detail the variational Poisson cohomology $\mathcal{H}_{K}^{k}$, and its $\mathbb{Z}$-graded Lie superalgebra structure, for a "hydrdynamic type" Hamiltonian operator, i.e. for $K(\partial)=S \partial$, where $S \in$ Mat $_{\ell \times \ell}(\mathcal{C})$ is nondegenerate and symmetric.

6.2. Translation invariant variational Poisson cohomology for $K=$ $S \partial$. As in the previous section, let $\mathcal{V}$ be a translation invariant normal algebra of differential functions, with field of constants $\mathcal{C}$ (which coincides with the field of quasiconstants). Let $S \in \operatorname{Mat}_{\ell \times \ell}(\mathcal{C})$ be nondegenerate and symmetric, and consider the Hamiltonian operator $K(\partial)=S \partial$.

For $k \geq-1$, we denote by $\Lambda^{k+1}$ the space of skewsymmetric $(k+1)$ linear forms on $\mathcal{C}^{\ell}$, i.e. the space of arrays $B=\left(b_{i_{0}, \ldots, i_{k}}\right)_{i_{0}, \ldots, i_{k} \in I}$, totally skewsymmetric with respect to permutations of the indices $i_{0}, \ldots, i_{k}$. For $k \geq 0$, we also denote by $\Lambda_{S}^{k+1}$ the space of arrays of the form $A=$ $\left(a_{j, i_{1}, \ldots, i_{k}}\right)_{j, i_{1}, \ldots, i_{k} \in I}$, which are skewsymmetric with respect to permutations of the indices $i_{1}, \ldots, i_{k}$, and which satisfy the equation

$$
\sum_{j \in I} s_{i_{0}, j} a_{j, i_{1}, i_{2} \ldots, i_{k}}=-\sum_{j \in I} a_{j, i_{0}, i_{2}, \ldots, i_{k}} s_{j, i_{1}} .
$$

Clearly, $\operatorname{dim}_{\mathcal{C}}\left(\Lambda_{S}^{k+1}\right)=\operatorname{dim}_{\mathcal{C}}\left(\Lambda^{k+1}\right)=\left(\begin{array}{c}\ell \\ k+1\end{array}\right)$ for every $k \geq-1$. For example, $\Lambda^{0}=\mathcal{C}, \Lambda_{S}^{1}=\Lambda^{1}=\mathcal{C}^{\ell}, \Lambda^{2}$ is the space of skewsymmetric $\ell \times \ell$ matrices over $\mathcal{C}$, and

$$
\Lambda_{S}^{2}=\left\{A \in \operatorname{Mat}_{\ell \times \ell}(\mathcal{C}) \mid A^{T} S+S A=0\right\}=\operatorname{so}(\ell, S) .
$$

Given $A=\left(a_{j, i_{0}, \ldots, i_{k}}\right)_{j, i_{0}, \ldots, i_{k} \in I} \in \Lambda_{S}^{k+2}$, we denote

$$
u A=\left(\sum_{j \in I} u_{j} a_{j, i_{0}, \ldots, i_{k}}\right)_{i_{0}, \ldots, i_{k} \in I} \in W_{k}^{v a r} .
$$


Let $\mathcal{A}^{\bullet}=\bigoplus_{k=-1}^{\infty} \mathcal{A}^{k}$, where

$$
\mathcal{A}^{k}=\Lambda^{k+1} \oplus\left\{u A \mid A \in \Lambda_{S}^{k+2}\right\} \subset W_{k}^{v a r}, k \geq-1 .
$$

Theorem 6.3. Let $\mathcal{V}$ be trnslation invariant normal algebra of differential functions, and let $K(\partial)=S \partial$, where $S$ is a symmetric nondegenerate $\ell \times \ell$ matrix over $\mathcal{C}$. Then:

(a) $\mathcal{A}^{\bullet}$ is a subalgebra of the $\mathbb{Z}$-graded Lie superalgebra $\mathcal{Z}_{K}^{\bullet}(\mathcal{V})$, complementary to the ideal $\mathcal{B}_{K}^{\bullet}(\mathcal{V})$. In particular, we have the following decomposition of $\mathcal{Z}_{K}^{k}$ in a direct sum of vector spaces over $\mathcal{C}$ :

$$
\mathcal{Z}_{K}^{k}=\mathcal{A}^{k} \oplus \mathcal{B}_{K}^{k}
$$

(b) We have an isomorphism of $\mathbb{Z}$-graded Lie superalgebras (cf. Secction 2.2):

$$
\mathcal{H}_{K}^{\bullet}(\mathcal{V})=\mathcal{A}^{\bullet} \simeq \widetilde{H}(\ell+1, \widetilde{S}),
$$

where $\widetilde{S}$ is the $(\ell+1) \times(\ell+1)$ matrix obtained from $S$ by adding a zero row and column. In particular, $\operatorname{dim}_{\mathcal{C}}\left(\mathcal{H}_{K}^{k}\right)=\left(\begin{array}{l}l+1 \\ k+2\end{array}\right)$.

Proof. For $B \in \Lambda^{k+1}$, we obviously have $\delta_{K} B=0$. Moreover, it is immediate to check, using the formula (3.19) for $\delta_{K}$, that, if $A \in \Lambda_{S}^{k+2}$, then $\delta_{K}(u A)=$ 0 . Hence, $\mathcal{A}^{k} \subset \mathcal{Z}_{K}^{k}$ for every $k \geq-1$. Next, we compute the box product (3.10) between two elements of $\mathcal{A}^{\bullet}$. Let $B \oplus u A \in \Lambda^{h+1} \oplus u \Lambda_{S}^{h+2}=\mathcal{A}^{h}$, and $D \oplus u C \in \Lambda^{k-h+1} \oplus u \Lambda_{S}^{k-h+2}=\mathcal{A}^{k-h}$. We have $B \square D=0, u A \square D=0$, moreover, $B \square u C \in \Lambda^{k+1} \subset \mathcal{A}^{k}$ and $u A \square u C \in u \Lambda_{S}^{k+2} \subset \mathcal{A}$ are given by

$$
\begin{aligned}
& (B \square u C)_{i_{0}, \ldots, i_{k}}=\sum_{\sigma \in \mathcal{S}_{h, k}} \operatorname{sign}(\sigma) \sum_{j \in I} b_{j, i_{\sigma(k-h+1)}, \ldots, i_{\sigma(k)}} c_{j, i_{\sigma(0)}, \ldots, i_{\sigma(k-h)},} \\
& (u A \square u C)_{i_{0}, \ldots, i_{k}}=\sum_{\sigma \in \mathcal{S}_{h, k}} \operatorname{sign}(\sigma) \sum_{i, j \in I} u_{i} a_{i, j, i_{\sigma(k-h+1)}, \ldots, i_{\sigma(k)}} c_{j, i_{\sigma(0)}, \ldots, i_{\sigma(k-h)}} .
\end{aligned}
$$

We thus conclude that $\mathcal{A}^{\bullet}=\bigoplus_{k \geq-1} \mathcal{A}^{k}$ is a subalgebra of the $\mathbb{Z}$-graded Lie superalgebra $\mathcal{Z}^{\bullet}(\mathcal{V}) \subset W^{\operatorname{var}}(\Pi \mathcal{V})$.

Since $\mathcal{S}_{-1, k+1}=\emptyset$, we have that $\mathcal{A}^{-1} \square \mathcal{A}^{\bullet}=0$. Moreover, $\mathcal{S}_{-1, k+1}=\{1\}$. Hence, for $d \oplus u C \in \mathcal{C} \oplus u \mathcal{C}^{\ell}=\mathcal{A}^{-1}$ and $B \oplus u A \in \Lambda^{k+1} \oplus u \Lambda_{S}^{k+2}=\mathcal{A}^{k}$, we have

$$
[B \oplus u A, d \oplus u C]=B \square(u C) \oplus(u A \square u C) \in \Lambda^{k} \oplus u \Lambda_{S}^{k+1}=\mathcal{A}^{k-1},
$$

with entries

$$
\begin{aligned}
& {[B, u C]_{i_{1}, \ldots, i_{k}}=(B \square u C)_{i_{1}, \ldots, i_{k}}=\sum_{j \in I} b_{j, i_{1}, \ldots, i_{k}} c_{j},} \\
& {[u A, u C]_{i_{1}, \ldots, i_{k}}=(u A \square u C)_{i_{1}, \ldots, i_{k}}=\sum_{i, j \in I} u_{i} a_{i, j, i_{1}, \ldots, i_{k}} c_{j} .}
\end{aligned}
$$

It is clear, from formula (6.8), that $[B \oplus u A, u C]=0$ for every $C \in \mathcal{C}^{\ell}$ if and only if $A=0$ and $B=0$. Hence $\mathcal{A}^{\bullet}$ is a transitive $\mathbb{Z}$-graded Lie superalgebra. 
Since $\left[\mathcal{B}_{K}^{k}, \mathcal{Z}_{K}^{-1}\right]=0$, it follows, in particular, that $\mathcal{A}^{k} \cap \mathcal{B}_{K}^{k}=0$ for every $k \geq-1$. Hence $\mathcal{A}^{k}$ coincides with its image in $\mathcal{H}_{K}^{k}(\mathcal{V})$, and $\mathcal{A}^{\bullet}$ can be viewed as a subalgebra of the $\mathbb{Z}$-graded Lie superalgebra $\mathcal{H}_{K}^{\bullet}(\mathcal{V})$. Therefore (by the Tartaglia-Pascal triangle) $\operatorname{dim}_{\mathcal{C}} \mathcal{H}_{K}^{k} \geq \operatorname{dim}_{\mathcal{C}} \mathcal{A}^{k}=\left(\begin{array}{l}\ell+1 \\ k+2\end{array}\right)$. Since, by (6.4), $\operatorname{dim}_{\mathcal{C}} \mathcal{H}_{K}^{k} \leq\left(\begin{array}{l}\ell+1 \\ k+2\end{array}\right)$, we conclude that all these inequalities are equalities, and that $\mathcal{H}^{\bullet}(\mathcal{V}) \simeq \mathcal{A}^{\bullet}$ are isomorphic $\mathbb{Z}$-graded Lie superalgebras.

To conclude, in view of Proposition 2.2, we need to prove that $\mathcal{A}^{\bullet}$ is the full prolongation of the pair $\left(\mathcal{C}^{\ell+1}, s o(\ell+1, \widetilde{S})\right.$, where $\widetilde{S}$ is the $(\ell+1) \times(\ell+1)$ matrix obtained adding a zero row and column to $S$. We have $\mathcal{C}^{\ell+1}=\mathcal{C} \oplus \mathcal{C}^{\ell}$, and

$$
s o(\ell+1, \widetilde{S})=\left\{\left(\begin{array}{cc}
0 & B^{T} \\
0 & A
\end{array}\right) \mid B \in \mathcal{C}^{\ell}, A \in \operatorname{so}(\ell, S)\right\} \simeq \mathcal{C}^{\ell} \oplus \operatorname{so}(\ell, S),
$$

with the Lie bracket of $B \oplus A \in \mathcal{C}^{\ell} \oplus s o(\ell, S)$ and $d \oplus C \in \mathcal{C} \oplus \mathcal{C}^{\ell}$ given by

$$
[B+A, d+C]=B \cdot C \oplus A C \in \mathcal{C} \oplus \mathcal{C}^{\ell} .
$$

By definition, we have $\mathcal{A}^{0}=\Lambda^{1} \oplus u \Lambda_{S}^{2}=\mathcal{C}^{\ell} \oplus u \cdot \operatorname{so}(\ell, S)$, and the action of $B \oplus u A \in \mathcal{C}^{\ell} \oplus u \cdot s o(\ell, S)$ on $d \oplus u C \in \mathcal{C} \oplus u \mathcal{C}^{\ell}=\mathcal{A}^{-1}$, given by (6.8), is $[B \oplus u A, d \oplus u C]_{i}=B \cdot C \oplus u A C$. Namely, in view of (6.9), it is induced by the natural action of $s o(\ell+1, \widetilde{S}) \simeq \mathcal{C}^{\ell} \oplus \operatorname{so}(\ell, S)$ on $\mathcal{C} \oplus \mathcal{C}^{\ell}$. Hence, $\mathcal{A}^{-1} \oplus \mathcal{A}^{0} \simeq\left(\mathcal{C} \oplus \mathcal{C}^{\ell}\right) \oplus\left(\mathcal{C}^{\ell} \oplus \operatorname{so}(n, S)\right)$. Since $\mathcal{A}^{\bullet}$ is a transitive $\mathbb{Z}$-graded Lie superalgebra, it is a subalgebra of the full prolongation of $\left(\mathcal{C}^{\ell+1}, s o(\ell+1, \widetilde{S})\right.$.

On the other hand, by Proposition 2.2 the full prolongation of $\left(\mathcal{C}^{\ell+1}, s o(\ell+\right.$ $1, \widetilde{S})$ is isomorphic to $\widetilde{H}(\ell+1, \widetilde{S})$, and $\operatorname{dim}_{\mathcal{C}} \widetilde{H}(\ell+1, \widetilde{S})=2^{\ell+1}-1=$ $\sum_{k \geq-1} \operatorname{dim}_{\mathcal{C}} \mathcal{A}^{k}$. Hence, $\mathcal{A}^{\bullet}$ must be isomorphic to $\widetilde{H}(\ell+1, \widetilde{S})$, as we wanted.

Corollary 6.4. Under the assumptions of Theorem 6.3, the essential variational cohomology $\mathcal{E H}_{K}^{\bullet}(\mathcal{V})$ is zero.

Proof. It immediately follows from the transitivity of the $\mathbb{Z}$-graded Lie superalgebra $\mathcal{H}_{K}^{\bullet}(\mathcal{V})$.

Remark 6.5. If $S$ is a nondegenerate, but not necessarily symmetric, $\ell \times \ell$ matrix, we still have an isomorphism of vector spaces $\mathcal{H}_{K}^{k} \simeq \mathcal{A}^{k}$, but $\mathcal{H}_{K}^{\bullet}(\mathcal{V})$ is not, in general, a Lie superalgebra.

Remark 6.6. The description of $\mathcal{H}_{K}^{\bullet}(\mathcal{V})$, as a vector space, for $K=S \partial$ with $S$ symmetric nondegenerate matrix over $\mathcal{C}$, agrees with the results of S.-Q. Liu and Y. Zhang [LZ11, LZ11pr.

\section{REFERENCES}

[BDSK09] A. Barakat, A. De Sole, and V.G. Kac, Poisson vertex algebras in the theory of Hamiltonian equations, Japan. J. Math. 4, (2009) 141-252.

[DMS05] L. Degiovanni, F. Magri, and V. Sciacca, On deformation of Poisson manifolds of hydrodynamic type Comm. Math. Phys. 253, (2005) no. 1, 1-24. 
[DSK06] A. De Sole, and V.G. Kac, Finite vs. affine $W$-algebras, Japan. J. Math. 1, (2006) 137-261.

[DSK09] A. De Sole, and V.G. Kac, Lie conformal algebra cohomology and the variational complex, Commun. Math. Phys. 292, (2009) 667-719.

[DSK11] A. De Sole, and V.G. Kac, The variational Poisson cohomology, (2011) arXiv:1106.0082,

[DSKW10] A. De Sole, V.G. Kac, and M. Wakimoto, On classification of Poisson vertex algebras, Transform. Groups 15 (2010), no. 4, 883-907.

[Dor93] I.Ya. Dorfman, Dirac structures and integrability of nonlinear evolution equations, Nonlinear Sci. Theory Appl. (John Wiley \& Sons, 1993) 176 pp.

[Get02] E.Getzler, A Darboux theorem for Hamiltonian operators in the formal calculus of variations, Duke Math. J. 111 (2002), no. 3, 535-560.

[GS64] V.W. Guillemin, S. Sternberg, An algebraic model of transitive differential geometry Bull. Amer. Math. Soc. 70 (1964) 16-47.

[Kac77] V.G. Kac, Lie superalgebras Advances in Math. 26 (1977), no. 1, 8-96.

[Kra88] I.S. Krasilshchik, Schouten brackets and canonical algebras, Lecture Notes in Math. 1334, (Springer Verlag, New York 1988).

[Kup80] B.A. Kupershmidt, Geometry of jet bundles and the structure of Lagrangian and Hamiltonian formalisms, in Geometric methods in Mathematical Physics, Lecture Notes in Math. 775, (Springer Verlag, New York 1980) 162-218.

[Lic77] A. Lichnerowicz, Les varietes de Poisson et leur algebres de Lie associees, J. Diff. Geom. 12, (1977) 253-300.

[LZ11] S.-Q. Liu, and Y. Zhang, Jacobi structures of evolutionary partial differential equations, Adv. Math. 227 (2011), 73-130.

[LZ11pr] S.-Q. Liu, and Y. Zhang, private communication, Beijing, June 2011.

[Mag78] F. Magri, A simple model of the integrable Hamiltonian equation, J. Math. Phys. 19, (1978) 129-134.

[Olv87] P.J. Olver, BiHamiltonian systems, in Ordinary and partial differential equations, Pitman Research Notes in Mathematics Series 157, (Longman Scientific and Technical, New York 1987) 176-193. 